\title{
Graft-versus-host disease propagation depends on increased intestinal epithelial tight junction permeability
}

\author{
Sam C. Nalle, ${ }^{1}$ Li Zuo,, ${ }^{2,3}$ Ma. Lora Drizella M. Ong, ${ }^{2}$ Gurminder Singh, ${ }^{1,2}$ Alicia M. Worthylake, ${ }^{2}$ Wangsun Choi, ${ }^{2}$ \\ Mario Cabrero Manresa, ${ }^{2}$ Anna P. Southworth, ${ }^{2}$ Karen L. Edelblum, ${ }^{1,4}$ Gregory J. Baker, ${ }^{5}$ Nora E. Joseph, ${ }^{1}$ Peter A. Savage, ${ }^{1}$ \\ and Jerrold R. Turner ${ }^{1,2}$
}

'Department of Pathology, The University of Chicago, Chicago, Illinois, USA. '2Department of Pathology, Brigham and Women's Hospital and Harvard Medical School, Boston, Massachusetts, USA. ${ }^{3}$ Anhui Medical University, Hefei, Anhui, China. " Department of Pathology \& Laboratory Medicine, Center for Inflammation and Immunity, Rutgers New Jersey Medical School, Cancer Center, Newark, New Jersey, USA. ${ }^{5}$ Laboratory of Systems Pharmacology, Harvard Medical School, Harvard Program in Therapeutic Science, Boston, Massachusetts, USA.

\begin{abstract}
Graft-versus-host disease (GVHD) is a complication of hematopoietic stem cell transplantation (HSCT) that affects multiple organs. GVHD-associated intestinal damage can be separated into two distinct phases, initiation and propagation, which correspond to conditioning-induced damage and effector T cell activation and infiltration, respectively. Substantial evidence indicates that intestinal damage induced by pretransplant conditioning is a key driver of GVHD initiation. Here, we aimed to determine the impact of dysregulated intestinal permeability on the subsequent CVHD propagation phase. The initiation phase of CVHD was unchanged in mice lacking long MLCK (MLCK210), an established regulator of epithelial tight junction permeability. However, MLCK210-deficient mice were protected from sustained barrier loss and exhibited limited GVHD propagation, as indicated by reduced histopathology, fewer $\mathrm{CD}^{+}$effector T cells in the gut, and improved overall survival. Consistent with these findings, intestinal epithelial MLCK210 expression and enzymatic activity were similarly increased in human and mouse GVHD biopsies. Intestinal epithelial barrier loss mediated by MLCK210 is therefore a key driver of the GVHD propagation. These data suggest that inhibition of MLCK210-dependent barrier regulation may be an effective approach to limiting CVHD progression.
\end{abstract}

\section{Introduction}

Allogeneic hematopoietic stem cell transplantation (HSCT) is used in the treatment of neoplastic and immune disorders. Graftversus-host disease (GVHD), in which donor-derived $\mathrm{T}$ cells target recipient alloantigens to cause tissue pathology, is a common, and frequently severe, complication of HSCT (1). Tissue damage that occurs as a result of pretransplant conditioning is an important factor in GVHD initiation $(2,3)$, and we have demonstrated that this primarily reflects a requirement for intestinal injury and associated barrier loss (4). Thus, although conditioning-induced damage typically resolves before GVHD is clinically apparent, the two processes are closely linked.

Although efforts to reduce conditioning-associated intestinal damage for prevention of GVHD initiation have shown some efficacy, their utility is limited by the fact that intense antineoplastic therapies and bone marrow ablation are necessary for successful HSCT $(2,3)$. We therefore focused on the lesswell-defined mechanisms by which barrier loss promotes ongoing attack of recipient tissues, i.e., GVHD propagation, which is associated with local $\mathrm{T}$ cell recruitment and apoptosis of intestinal crypt epithelial cells $(5,6)$. Identification of these mech-

Authorship note: LZ and MLDMO contributed equally to this work. Conflict of interest: The authors have declared that no conflict of interest exists. License: Copyright 2019, American Society for Clinical Investigation. Submitted: November 7, 2017; Accepted: November 27, 2018. Reference information: J Clin Invest. 2019;129(2):902-914. https://doi.org/10.1172/JCI98554. anisms and means to prevent GVHD propagation could have tremendous therapeutic potential (7-9).

Sustained intestinal barrier loss has been proposed as a contributor to GVHD propagation based on the observation that the degree of intestinal barrier loss correlates with disease severity in patients and mice with GVHD (4, 6, 8-13). However, it remains unclear whether this increased permeability is a consequence of disease or a primary driver of disease propagation. This difficulty in separating cause from effect has prevented detailed characterization of intestinal barrier dysfunction as a contributor to GVHD pathogenesis. To resolve this, we focused on defining mechanisms and consequences of intestinal barrier loss during GVHD propagation.

Here, our analysis of human GVHD biopsies revealed elevated expression and enzymatic activity of long myosin light chain kinase (MLCK210), a well-defined regulator of tight junction permeability (2,14-16), suggesting that MLCK210-dependent alterations in barrier function may drive GVHD propagation. Consistent with this hypothesis, mechanistic studies in mice revealed that MLCK210 deficiency in recipient mice reduced GVHD propagation. Our studies demonstrate a requirement for MLCK210dependent intestinal permeability increases during the propagation phase and indicate that intestinal epithelial MLCK210 may be an accessible target for limiting GVHD incidence and severity.

\section{Results}

MLCK210 expression and activity are increased in human GVHD. In an effort to understand GVHD propagation, we analyzed intestinal biopsies from patients with GVHD for common hallmarks of 
A

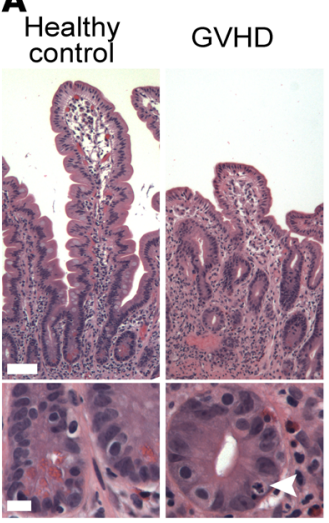

B

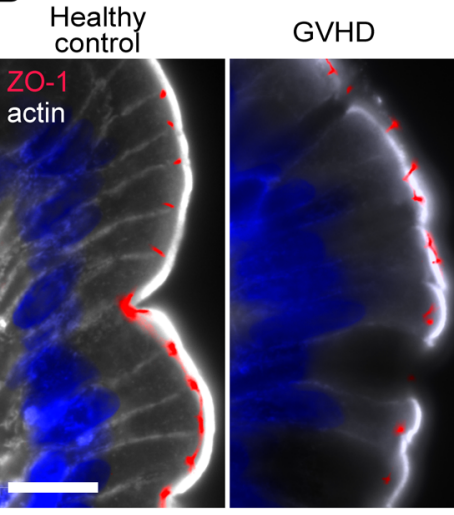

C
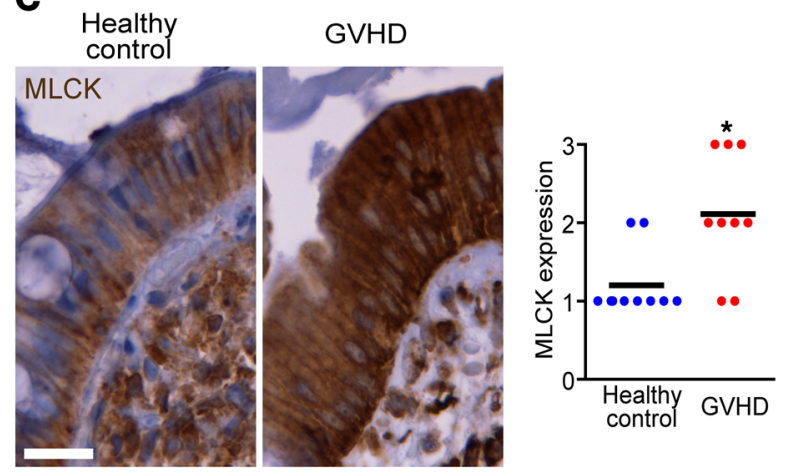

D
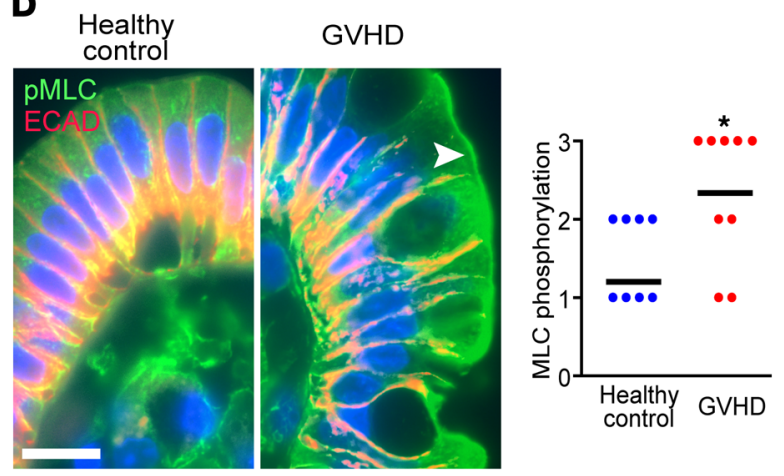

Figure 1. MLCK210 expression and myosin light chain phosphorylation are increased within the intestinal epithelium of patients with GVHD. (A) Representative histopathology of human small intestinal biopsies. Arrowhead denotes an apoptotic cell. Scale bars: $50 \mu \mathrm{m}$ (top), $10 \mu \mathrm{m}$ (bottom). (B) Human small intestinal biopsies from healthy controls (left) or CVHD patients (right) were immunostained for ZO-1 (red), $\beta$-actin (white), and Hoechst 33342 (blue). Scale bar: $10 \mu \mathrm{m}$. Representative images are shown. (C) Biopsies from healthy controls (left) or GVHD patients (right) were immunostained for MLCK210 (brown), counterstained with hematoxylin, and scored from 0 to 3 . Each point represents an individual patient. Representative images from $n=9$ patients and $n=8$ controls are shown. ${ }^{*} P<0.05$, Mann-Whitney $U$ test. Scale bar: $10 \mu \mathrm{m}$. (D) Biopsies from healthy controls (left) or GVHD patients (right) were immunostained for phosphorylated myosin light chain (pMLC, green) and E-cadherin (ECAD, red). Arrowhead denotes MLC phosphorylation at the perijunctional actomyosin ring. Stains were scored from 0 to 3 , with each point representing an individual patient. Representative images from $n=9$ patients and $n=8$ controls are shown. ${ }^{*} P<0.05$, Mann-Whitney $U$ test. Scale bar: $10 \mu \mathrm{m}$. intestinal damage and altered intestinal permeability. Although patients with GVHD have increased intestinal permeability (17), epithelial damage is limited to scattered apoptotic crypt epithelial cells in most cases (Figure 1A) (18-20). Our analyses therefore focused on patients with epithelial apoptosis but without greater degrees of epithelial damage. Tight junction and epithelial structure within intestinal biopsies from these GVHD patients were generally intact, as indicated by unaltered distribution of the tight junction protein zonula occludens-1 (ZO-1) and actin relative to healthy control subjects (Figure 1B). We therefore asked whether expression of MLCK210, a principal regulator of the tight junction leak pathway, was increased in small intestinal biopsies from patients with GVHD relative to healthy controls (Figure 1C). GVHD biopsies exhibited increased MLCK210 expression, and enhanced enzymatic activity, as indicated by increased myosin II regulatory light chain (MLC) phosphorylation (Figure 1D). These data are consistent with the hypothesis that MLCK210-mediated changes in tight junction permeability are associated with GVHD pathogenesis in human subjects.

Intestinal epithelial MLCK210 expression and activity are increased after allogeneic bone marrow transplantation in mice. We previously developed a minor antigen mismatch GVHD model that recapitulates human GVHD (4). In this bone marrow transplant (BMT) model, transfer of donor bone marrow cells together with mature splenocytes from mice of the $129 \mathrm{~S} 6$ strain (herein, 129) drives GVHD following infusion into irradiated C57BL/6J (B6) recipient mice. Of note, the 129 and B6 mouse strains are MHC matched ( $\mathrm{H}-2^{\mathrm{b}}$ haplotype), but harbor minor antigen mismatches that trigger allorecognition of recipient $\mathrm{B} 6$ cells by donor $129 \mathrm{~T}$ cells. This system recapitulates many features of human GVHD after HSCT, which is commonly HLA matched. For example, GVHD propagation is relatively slow in this mouse model, with appearance of clinical features on day 21 (d21) after BMT. This allows unequivocal separation of GVHD propagation from initiation-inducing damage caused by conditioning, as the latter is healed by d14 after irradiation (4). Histological examination on d14 after BMT showed that epithelial damage was limited to rare apoptotic crypt cells after allogeneic BMT $(129 \rightarrow$ B6) but not syngeneic BMT (B6 $\rightarrow$ B6) (Figure 2A). As in human GVHD, jejunal epithelial MLCK expression (Figure 2, B and D) and activity (Figure 2, C and D) were increased after allogeneic BMT. Disease initiation in this model is characterized by IFN- $\gamma$, IL- $1 \beta$, and TNF upregulation within jejunal mucosa after allogeneic, but not syngeneic, BMT (Figure 2E), consistent with previous work showing that TNF and IL-1 $\beta$ signaling induce MLCK210 upregulation (21-23). Thus, this model recapitulates MLCK210 upregulation in human disease and can be utilized to assess the impact of MLCK210dependent barrier regulation on GVHD pathogenesis.

Experimental GVHD-associated barrier loss requires intestinal epithelial MLCK210. Epithelial MLCK210 regulates tight junction permeability in two distinct ways. Modest MLCK210 activation, such as that induced by physiological stimuli, increases paracellular permeability to molecules with diameters up to $\sim 8 \AA(24,25)$. In contrast, activation of MLCK210 by pathologic stimuli, such as TNF, increases tight junction permeability to larger molecules, including those with diameters of $28 \AA$ and $70 \AA(2,14,26-28)$. To assess these distinct modes of MLCK210-dependent barrier loss, we measured intestinal 

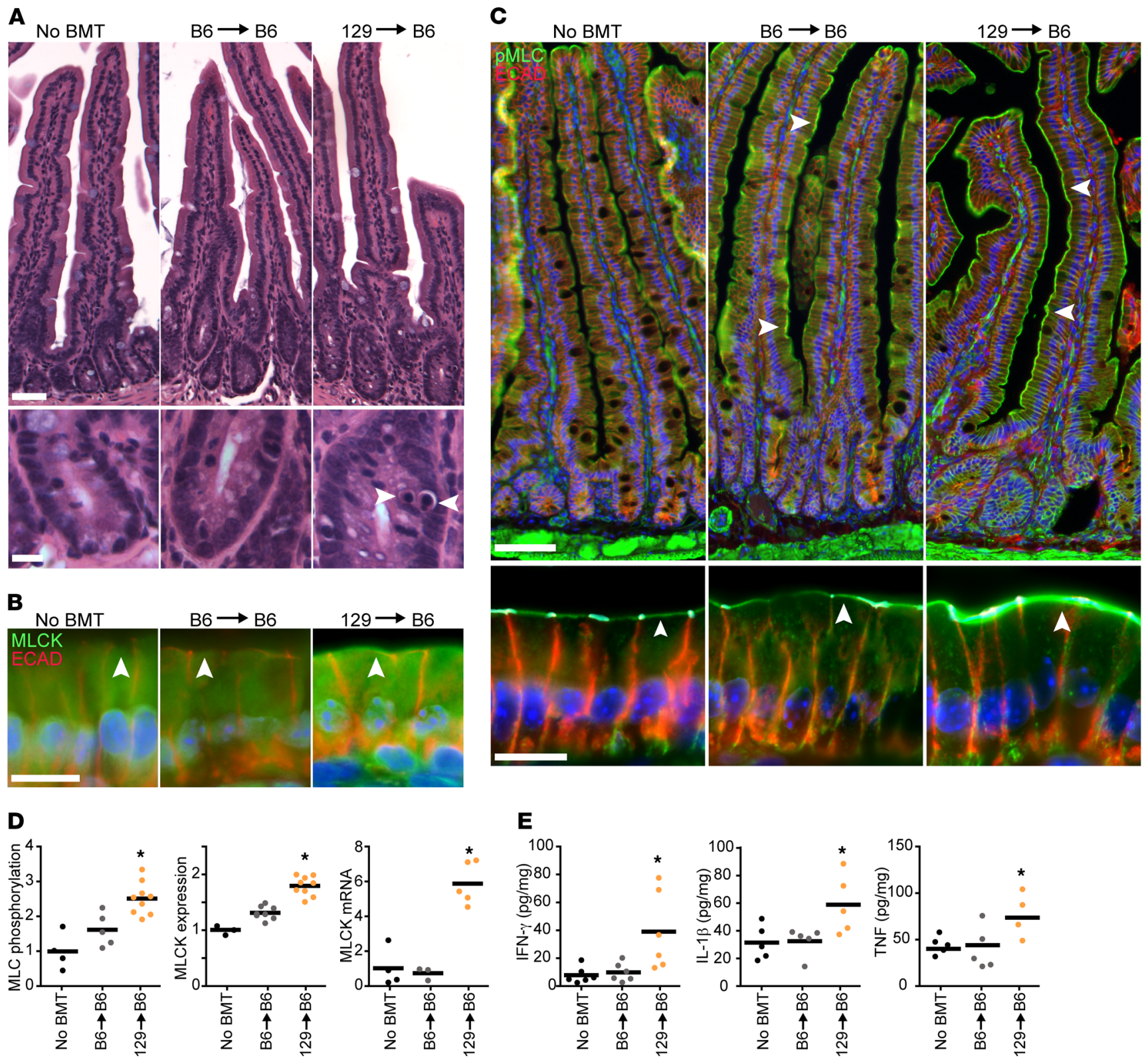

\section{$\mathbf{E}$}
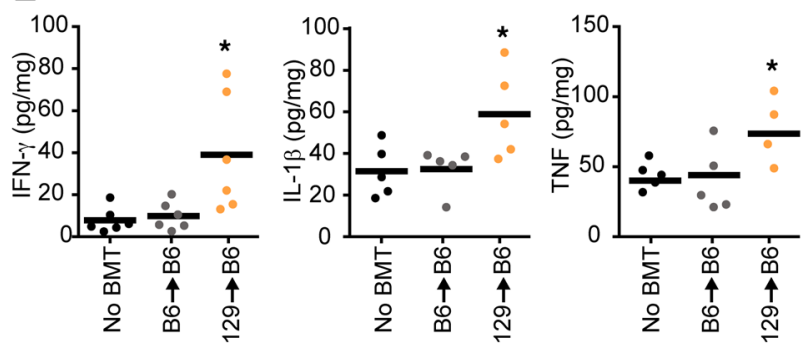

Figure 2. MLCK210 expression and activity as well as cytokines associated with MLCK210 upregulation are elevated in minor mismatch experimental GVHD. B6 WT recipients were lethally irradiated followed by a syngeneic (B6) or allogeneic (129) BMT. Mice were sacrificed 14 days after BMT. (A) Representative histopathology of the small intestine. Arrowheads denote apoptotic epithelial cells. Scale bars: $50 \mu \mathrm{m}$ (top), $10 \mu \mathrm{m}$ (bottom). (B) Jejunal segments were immunostained for MLCK210 (green) and E-cadherin (red). Arrowheads indicate the location of the perijunctional actomyosin ring. Images are representative of $>3$ independent experiments. Quantitative analysis is shown in D. Scale bar: $10 \mu \mathrm{m}$. (C) Jejunal segments were immunostained for phosphorylated myosin light chain (pMLC, green) and E-cadherin (red). Arrowheads indicate the location of the perijunctional actomyosin ring. Images are representative of $>3$ independent experiments. Quantitative analysis is shown in D. Scale bars: $50 \mu \mathrm{m}$ (top), $10 \mu \mathrm{m}$ (bottom). (D) MLCK210 expression and MLC phosphorylation were determined morphometrically. Each point represents an average of 4 fields from one segment of tissue from a single mouse. Two segments were analyzed per mouse. Data are normalized to the mean of mice that did not receive BMT. MLCK210 mRNA was determined by quantitative PCR ( $q P C R$ ) in purified epithelial cells. Each point represents an individual mouse. Data are normalized to the mean of mice that did not receive BMT. ${ }^{*} P<0.05$, 2-tailed $t$ test (B6 $\rightarrow$ WT vs. $129 \rightarrow W T$ ). (E) Jejunal cytokines were determined by ELISA. Each point represents an individual mouse. ${ }^{*} P<0.05$, 2-tailed $t$ test $(\mathrm{B} 6 \rightarrow \mathrm{WT}$ vs. $129 \rightarrow \mathrm{WT})$.

permeability of fluorescein and 4-kDa dextran (Figure 3A), which have diameters of $8 \AA$ and $28 \AA$, respectively, in WT and MLCK210deficient $\left(\mathrm{MLCK}^{--}\right)$mice on the B6 background that received a BMT from WT 129 donors. The $\mathrm{MLCK}^{-/-}$mice lack MLCK210 but display normal expression of the shorter, smooth muscle MLCK protein that is derived from the same $M y l k$ gene $(2,29)$.
On d14 after BMT, increased intestinal fluorescein permeability was relatively muted in $\mathrm{MLCK}^{-/-}$mice compared with WT controls (Figure 3B). Increased permeability to $4-\mathrm{kDa}$ dextran was not detected on d14, but it was significantly reduced in $\mathrm{MLCK}^{-1}$, relative to WT, on $\mathrm{d} 35$ (Figure 3, C and D). These permeability changes corresponded with increased intestinal epithelial MLC 
A

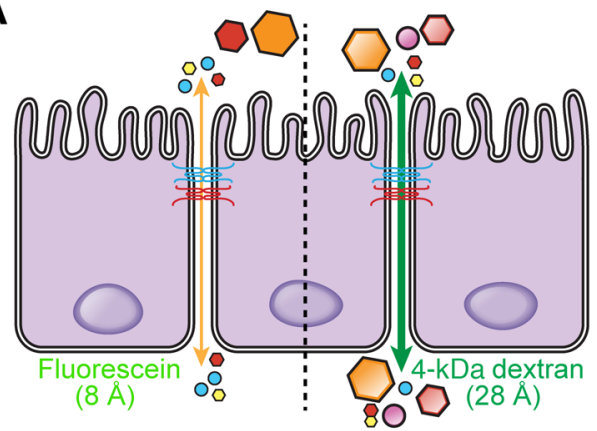

E
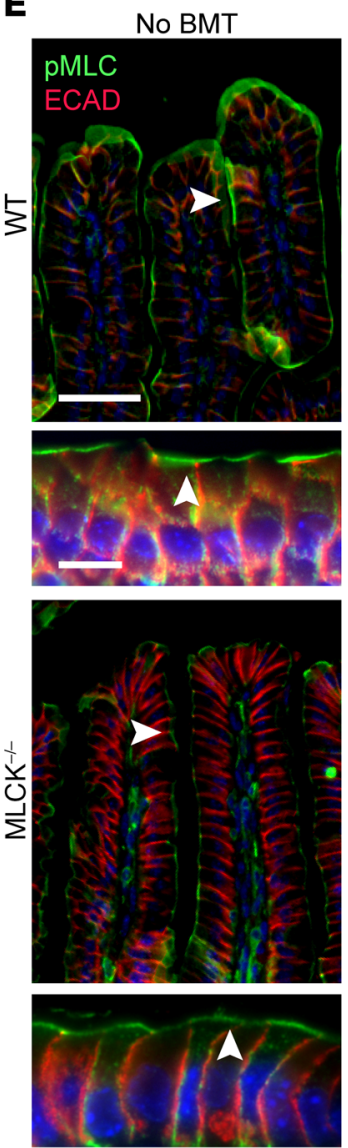

B

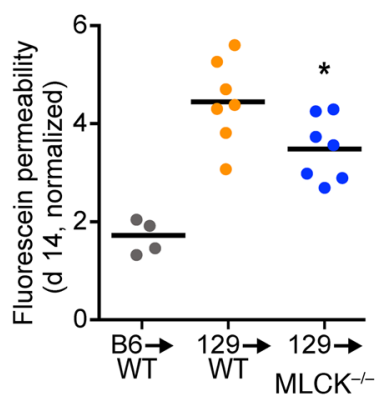

C

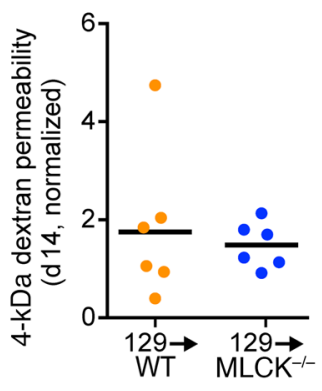

$\mathbf{F}$

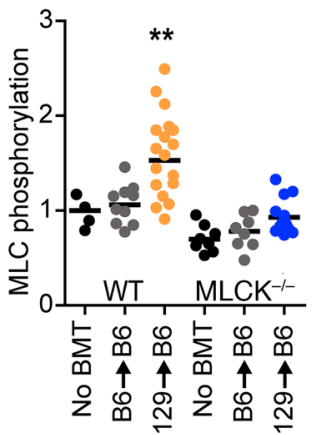

D

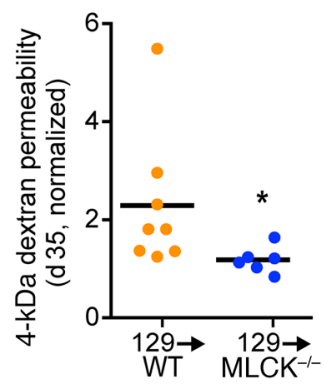

G

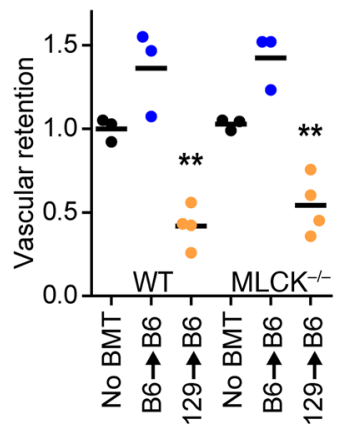

H
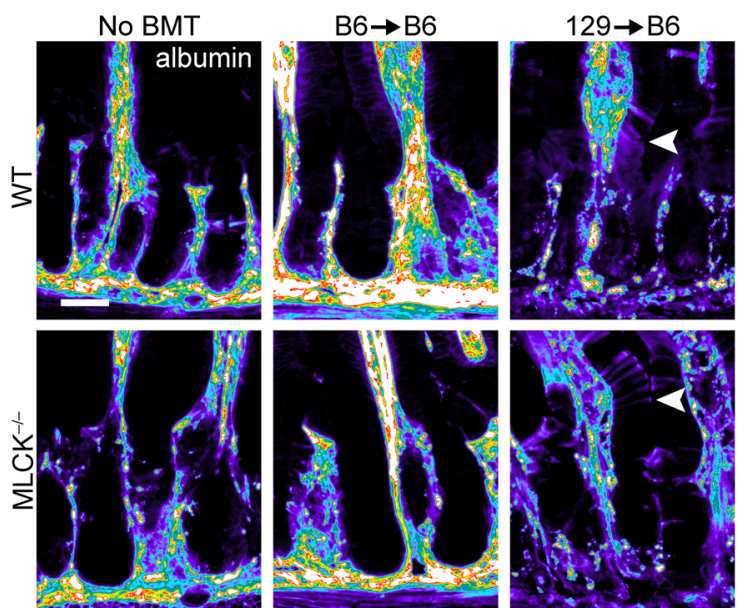

Figure 3. Allogeneic BMT fails to induce barrier dysfunction in $\mathbf{M L C K}^{-/-}$mice. (A) Probes used to measure MLCK210-dependent barrier loss. (B) Intestinal permeability to fluorescein was evaluated on d14 after syngeneic (B6 $\rightarrow$ WT) or allogeneic (129 $\rightarrow$ WT or MLCK ${ }^{-/-}$) BMT. Values are normalized to the mean of WT mice that did not receive BMT. Each point represents an individual mouse. ${ }^{*} P<0.05,2$-tailed $t$ test. (C and $\left.\mathbf{D}\right)$ Intestinal permeability to 4 - $k$ Da dextran on d14 (C) or d35 (D) after BMT, normalized to the mean of WT mice without BMT. Each point represents an individual mouse. ${ }^{*} P<0.05$, 2 -tailed $t$ test. (E and F) Jejunum harvested on d35 and immunostained for phosphorylated myosin light chain (pMLC, green) and E-cadherin (ECAD, red). Arrowheads denote perijunctional actomyosin ring. Note the preservation of myosin light chain phosphorylation within villus smooth muscle in MLCK ${ }^{\prime-}$ mice. Each point represents the average of 4 fields from one segment of tissue; 2 segments were analyzed per mouse. Data are normalized to the mean of WT mice without BMT. Scale bars: $50 \mu \mathrm{m}$ (top), $10 \mu \mathrm{m}$ (bottom). ${ }^{* *} P<0.01$, ANOVA with Bonferroni's correction (vs. all other conditions). (G and H) 35 days after BMT, mice were injected with Alexa Fluor 647-BSA and sacrificed 30 minutes later. Confocal imaging demonstrates bright intravascular signal, indicative of retained dye, in WT and $\mathrm{MLCK}^{-/-}$mice without BMT or after syngeneic $(\mathrm{B} 6 \rightarrow \mathrm{B} 6) \mathrm{BMT}$. In contrast, intravascular signal is markedly diminished in both WT and $\mathrm{MLCK}^{-1-}$ mice after allogeneic $\left(129 \rightarrow\right.$ B6) BMT. Note the sharp paracellular pattern in MLCK ${ }^{-1-}$ mice, consistent with preservation of the tight junction barrier, relative to the diffuse signal over the epithelium of WT mice (arrowheads). Scale bar: $25 \mu \mathrm{m}$. Each point represents an average of 6 regions of lamina propria from one mouse. Data are normalized to mean of WT mice without BMT. ${ }^{* *} P<0.01$, ANOVA with Bonferroni's correction (vs. other conditions for each genotype; matched conditions across genotypes were not significantly different). 
A

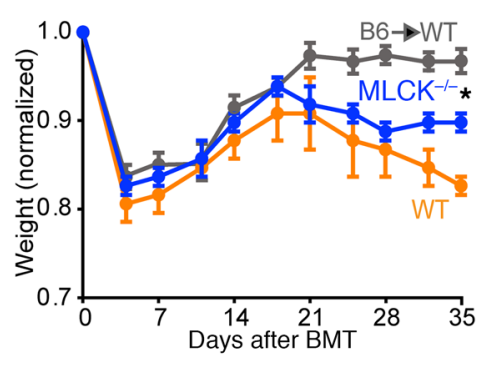

D

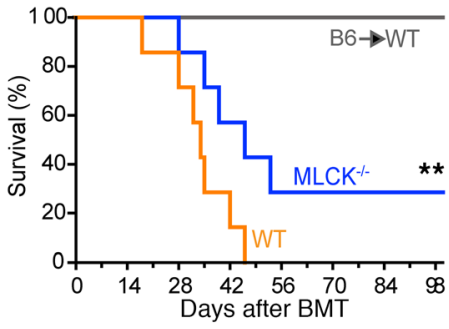

$\mathbf{F}$
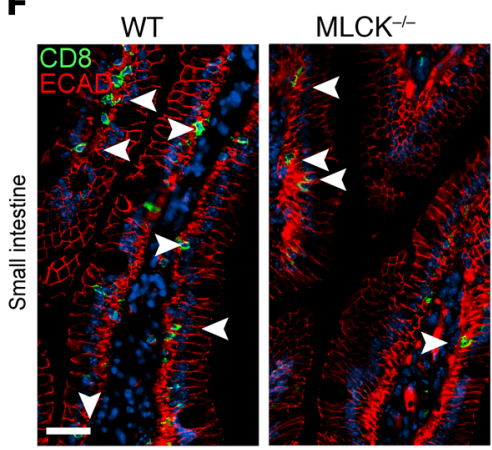

B

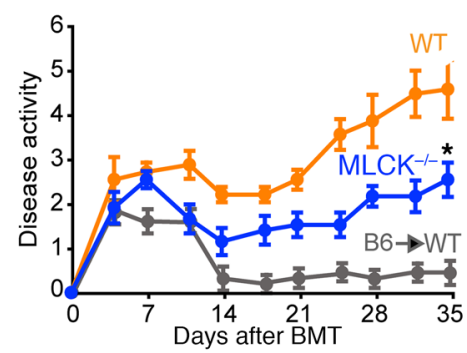

E

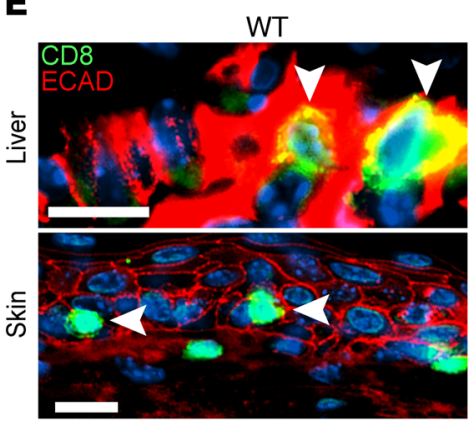

C

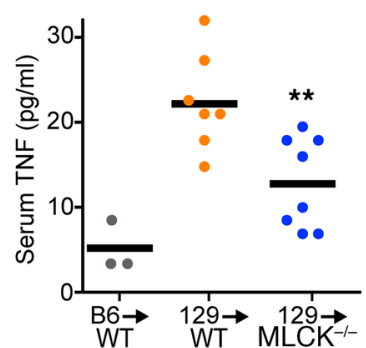

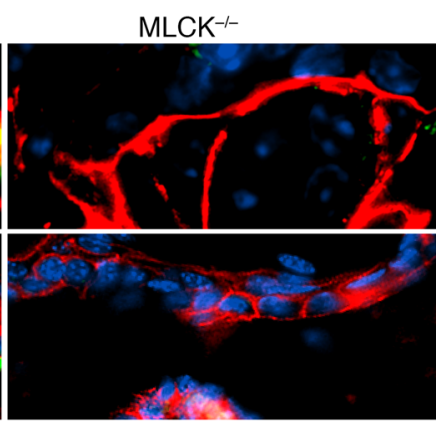

G
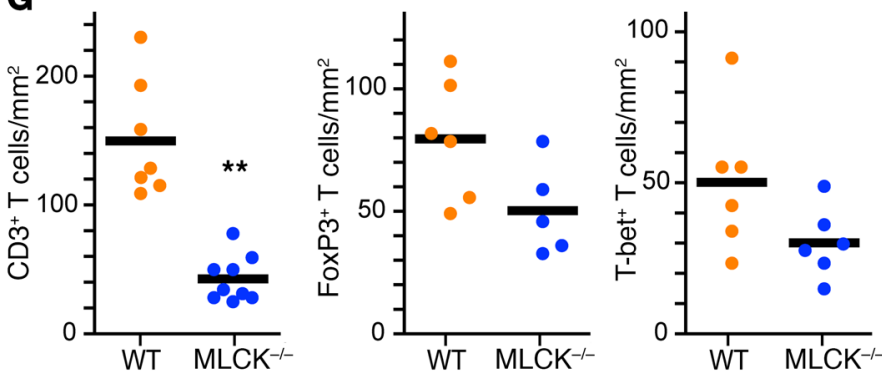

Figure 4. GVHD severity is markedly reduced in $\mathrm{MLCK}^{-/-}$mice. B6 WT or $\mathrm{MLCK}^{-1-}$ recipients were lethally irradiated, followed by a syngeneic (B6) or allogeneic (129) BMT. (A) Relative weight and (B) disease activity scores $\left(n=8-12\right.$ /group). ${ }^{*} P<0.05$, 2-tailed $t$ tests on d35 (129 $\rightarrow$ WT vs. 129 $\rightarrow$ MLCK ${ }^{-1}$ ). (C) Serum TNF as determined by ELISA, 35 days after BMT. Each point represents an individual mouse. ${ }^{* *} P<0.01,2$-tailed $t$ test (129 $\rightarrow$ WT vs. 129 $\rightarrow$ MLCK ${ }^{-I-}$ ). (D) Survival ( $n=6-8$ /group). ${ }^{* *} P<0.01$, Kaplan-Meier log-rank test $\left(129 \rightarrow W T\right.$ vs. $129 \rightarrow$ MLCK $\left.^{-1}\right)$. (E and F) Liver, skin, and jejunum were harvested 35 days after BMT and sections immunostained for CD8 (green) and E-cadherin (ECAD, red). (E) Representative images of biliary and squamous (skin) epithelium from WT (left panels) and $\mathrm{MLCK}^{-1-}$ (right panels) mice are shown. Arrowheads denote infiltration by CD8 ${ }^{+}$cells. Scale bars: $20 \mu \mathrm{m}$. (F) Representative images of jejunum from WT (left) and MLCK ${ }^{-1-}$ (right) mice. Arrowheads denote direct contact of CD8 ${ }^{+}$cells with the epithelium. Scale bar: $50 \mu \mathrm{m}$. (C) Jejunum harvested 35 days after BMT was immunostained for CD3, CD4, Foxp3, and T-bet. Graphs show $\mathrm{CD3}^{+}, \mathrm{CD}^{+} \mathrm{Foxp}^{+}$, or $\mathrm{CD} 4^{+} \mathrm{T}_{\text {-bet }}{ }^{+} \mathrm{T}$ cells. Each point represents an individual mouse. ${ }^{* *} P<0.01,2$-tailed $t$ test.

phosphorylation between d14 and d35 after BMT in WT mice (Figure 3, E and F). This was particularly evident within the apical perijunctional actomyosin ring, which interfaces directly with the tight and adherens junctions (30). Increased epithelial MLC phosphorylation was not detected in $\mathrm{MLCK}^{-/}$mice (Figure 3, E and F). Thus, although several kinases, e.g., Rho and citron kinases, can phosphorylate MLC in other cell types (31), MLCK210 is responsible for increased intestinal epithelial MLC phosphorylation during GVHD progression. Notwithstanding previous studies indicating a role for MLCK210 in endothelial contraction and regulation of microvascular permeability $(32,33)$, there were no differences between WT and $\mathrm{MLCK}^{-1-}$ mice in terms of tissue permeability to intravenously injected albumin 35 days after BMT (Figure 3, G and H). Therefore, the genetic MLCK210 inhibition in this GVHD model limited MLC phosphorylation and paracellular permeability in intestinal epithelia but did not prevent increased microvascular permeability.

MLCK210-dependent processes contribute to GVHD progression. Intestinal epithelial tight junction barrier preservation in $\mathrm{MLCK}^{-/-}$ mice provides an opportunity to define the contributions of tight junction dysregulation to GVHD progression during the propagation phase. Weight loss (Figure 4A) and disease activity (Figure 4B) were similar in WT and $\mathrm{MLCK}^{-/-}$mice during the first week after minor mismatch BMT, indicating that radiation-induced damage in the initiation phase was not affected by MLCK210 deficiency. Weight loss of $\mathrm{MLCK}^{-1-}$ and WT recipients diverged on $\mathrm{d} 28$ after allogeneic BMT, and by $\mathrm{d} 35$ weight loss in WT mice was $40 \%$ greater than that in $\mathrm{MLCK}^{-/}$mice (Figure $4 \mathrm{~A}$ ). Disease activity of 
$\mathrm{MLCK}^{--}$and WT mice differed within 10 days of BMT, and this discrepancy increased as disease progressed (Figure 4B). Serum TNF levels were also markedly lower in $\mathrm{MLCK}^{-/}$mice (Figure $4 \mathrm{C}$ ). Further, long-term survival was improved in $\mathrm{MLCK}^{-/}$, compared with WT, after allogeneic BMT (Figure 4D). As an additional measure of disease progression, we assessed intestinal mucosal infiltration by $\mathrm{CD} 8^{+} \mathrm{T}$ cells, the major effectors of tissue damage in GVHD. There were fewer $\mathrm{CD}^{+} \mathrm{T}$ cells within the intestinal mucosa as well as in biliary and skin epithelium of $\mathrm{MLCK}^{-/-}$minor mismatch (129) $\mathrm{BMT}$ recipients relative to $\mathrm{WT}$ (Figure 4, $\mathrm{E}$ and $\mathrm{F}$ ), in line with the reduced histopathology at these sites in $\mathrm{MLCK}^{--}$mice. Further, $\mathrm{CD}^{+} \mathrm{T}$ cell infiltrate density in the small intestine was markedly reduced in $\mathrm{MLCK}^{-/}$, relative to WT (Figure 4G). Subset analyses failed to show significant differences in numbers of $\mathrm{CD}^{+}{ }^{+} \mathrm{Foxp} 3^{+}$ regulatory or $\mathrm{CD} 4^{+} \mathrm{T}-$ bet $^{+} \mathrm{Th} 1$-polarized skewed $\mathrm{T}$ cells in $\mathrm{MLCK}^{-1}$ versus WT BMT recipients (Figure $4 G$ ).

Cumulatively, these data demonstrate that MLCK210 contributes significantly to systemic GVHD propagation but has no measurable effect on the initiation phase. Although a role for MLCK210 in non-epithelial cell types, e.g., endothelia, cannot be completely ruled out $(29,34)$, the use of donor cells from WT mice in these studies excludes a role for MLCK210-dependent changes in immune and other bone marrow-derived cells as a potential explanation for reduced disease in $\mathrm{MLCK}^{-/-}$recipients.

Intestinal epithelial MLCK21O drives barrier loss and GVHD propagation. To verify that the disease amelioration observed in $\mathrm{MLCK}^{--} \mathrm{BMT}$ recipients was due to deletion of MLCK210 within intestinal epithelia, we asked whether the protection observed in $\mathrm{MLCK}^{-/}$mice could be reversed by crossing $\mathrm{MLCK}^{-/-}$mice with transgenic mice specifically expressing MLCK within the intestinal epithelium. The MLCK $^{\mathrm{Tg}}$ mice used express constitutively active MLCK under control of the intestinal epithelium-specific 9-kb villin promoter (35). Weight loss (Figure 5A), disease activity (Figure $5 \mathrm{~B}$ ), survival (Figure $5 \mathrm{C}$ ), cytolytic $\mathrm{CD} 8^{+}$granzyme $\mathrm{B}^{+} \mathrm{T}$ cell infiltration (Figure 5D), and histopathology (Figure 5, E and F) of both $\mathrm{MLCK}^{\mathrm{Tg}} \mathrm{MLCK}^{--}$and $\mathrm{MLCK}^{\mathrm{Tg}}$ mice were all similar to those of WT mice. In contrast, each of these features of GVHD was significantly ameliorated in $\mathrm{MLCK}^{-/-}$mice relative to WT, $\mathrm{MLCK}^{\mathrm{Tg}} \mathrm{MLCK}^{-/}$, or MLCK $^{\mathrm{Tg}}$ mice (Figure 5, A-F). In addition, the hallmark skin ulceration observed in advanced GVHD was similar in MLCK ${ }^{\mathrm{Tg}}$ and WT mice, while this characteristic was absent and fur texture markedly less perturbed in $\mathrm{MLCK}^{-/}$mice (Figure $5 \mathrm{G}$ ).

To better define the impact of MLCK-dependent barrier regulation on GVHD propagation, we assessed overall disease severity on $\mathrm{d} 35$ as a function of barrier loss on $\mathrm{d} 14$ after BMT. When all 4 genotypes that received allogeneic BMT as well as mice that received syngeneic BMT were considered, there was a strong correlation between ultimate disease severity and permeability increases that occurred much earlier (Figure $5 \mathrm{H} ; r=0.75, P<0.01$ ). As a whole, these results indicate that disease propagation and cumulative GVHD severity are driven by MLCK210-dependent regulation of intestinal epithelial permeability.

MLCK210 inhibition reduces GVHD in a major antigen mismatch model. In order to test the role of MLCK210 in a separate GVHD model, we injected irradiated $\mathrm{B} 6$ recipients $\left(\mathrm{H}-\mathrm{2}^{\mathrm{b}}\right.$ haplotype) with bone marrow and splenocytes from BALB/c donors ( $\mathrm{H}-2^{\mathrm{d}}$ haplotype). In this pairing the donor and recipient are MHC mismatched, leading to substantial allorecognition of recipient antigen by donor immune cells. This results in a robust graft-versus-host response and rapid GVHD progression. As in minor mismatch GVHD, skin ulcerations (Figure 6A), histopathology (Figure 6, B and C), and disease activity (Figure 6D) were all improved in $\mathrm{MLCK}^{-/-}$, relative to WT, recipients. Despite protection, $\mathrm{d} 35$ disease activity scores of $\mathrm{MLCK}^{-/-}$mice receiving major mismatch BMT were substantially greater than those of $\mathrm{MLCK}^{-/-}$mice after minor mismatch BMT. The greater severity of major mismatch GVHD was also apparent in survival of WT mice; $40 \%$ survived to $\mathrm{d} 35$ after $\mathrm{BALB} / \mathrm{c} \rightarrow \mathrm{B} 6 \mathrm{BMT}$ relative to $\sim 70 \%$ after $129 \rightarrow$ B6 BMT. Nevertheless, survival of MLCK $^{-1-}$ mice on day 35 after major mismatch BMT was more than twice that of WT mice (Figure 6E).

Similar to minor mismatch disease, major mismatch GVHD was associated with marked increases in intestinal epithelial MLCK210 expression (Figure 6F) and intestinal epithelial MLC phosphorylation in WT mice (Figure 6, G and H). In contrast, no increases in MLC phosphorylation were detected in intestinal epithelia of $\mathrm{MLCK}^{-/-}$mice (Figure 6, G and H). Finally, lamina propria infiltration by $\mathrm{CD}^{+}$granzyme $\mathrm{B}^{+} \mathrm{T}$ cells was significantly greater in WT, relative to $\mathrm{MLCK}^{-/}$, mice after major mismatch BMT (Figure 6I). Taken together, these results demonstrate that MLCK210 deletion limits propagation of major antigen mismatch GVHD and provides further evidence that intestinal epithelial MLCK210 is a critical regulator of GVHD pathogenesis.

MLCK210-dependent intestinal epithelial tight junction barrier loss is associated with polyclonal cytolytic $T$ expansion within mesenteric lymph nodes. While the data above indicate a key role for MLCK210-dependent intestinal paracellular permeability increases in GVHD propagation, they do not explain how intestinal barrier loss is translated to systemic disease. To better assess immune aspects of disease progression, we developed an antigenspecific GVHD model. We used OVA as a model antigen, as the minor antigen(s) responsible for allorecognition have not been defined in the $129 \rightarrow \mathrm{B} 6$ model (36). WT and $\mathrm{MLCK}^{-1-} \mathrm{B} 6$ mice expressing membrane-bound OVA ( $\mathrm{mOVA}^{\mathrm{Tg}}$ and $\mathrm{mOVA}^{\mathrm{Tg}} \mathrm{MLCK}^{-/}$) were used as BMT recipients. For these studies, WT B6 bone marrow and splenocytes were supplemented with a minor fraction of splenocytes from congenically disparate (CD90.1) B6 OT-I mice, which express a transgenic $\mathrm{T}$ cell receptor reactive to the OVA peptide SIINFEKL in the context of $\mathrm{H}-2 \mathrm{~K}^{\mathrm{b}}$ (37). GVHD developed in $\mathrm{mOVA}^{\mathrm{Tg}}$ BMT recipients, but not in WT B6 recipients lacking membrane-bound OVA expression, confirming that the OVA peptide serves as a minor mismatch antigen rendering recipient mice susceptible to GVHD (Figure 7, A and B). When $\mathrm{mOVA}^{\mathrm{Tg}}$ and $\mathrm{mOVA}^{\mathrm{Tg}} \mathrm{MLCK}^{-/-} \mathrm{BMT}$ recipients were compared, the kinetics with which weight and disease activity scores diverged were similar to that in the 129 B6 allogeneic BMT model. Characteristic histopathological features of GVHD were evident in mOVA $^{\mathrm{Tg}} \mathrm{BMT}$ recipients but were significantly decreased in $\mathrm{mOVA}^{\mathrm{Tg}} \mathrm{MLCK}^{-1-}$ recipients (Figure 7C). Finally, consistent with the results in $129 \rightarrow$ B6 allogeneic BMT, the density of $\mathrm{CD}^{+} \mathrm{T}$ cells within the small intestine of $\mathrm{mOVA}^{\mathrm{Tg}} \mathrm{BMT}$ recipients was far greater than that in $\mathrm{mOVA}^{\mathrm{Tg}}$ $\mathrm{MLCK}^{-1-}$ recipients (Figure 7D). Thus, MLCK210 deletion in this antigen-specific model limits GVHD propagation and recapitulates the effects seen in minor and major antigen mismatch models. 
A

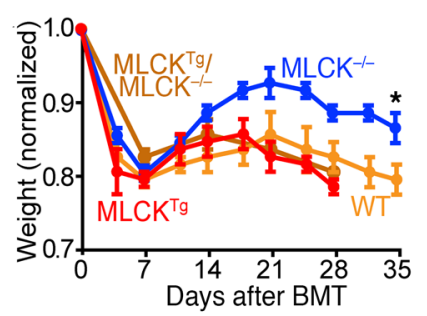

B

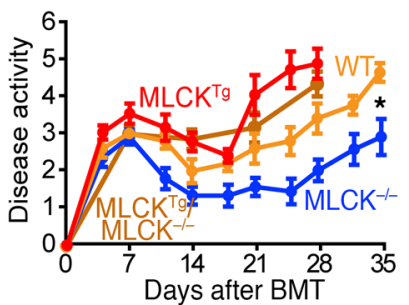

C

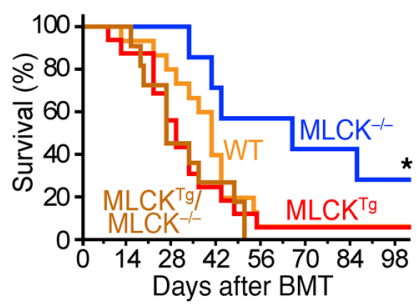

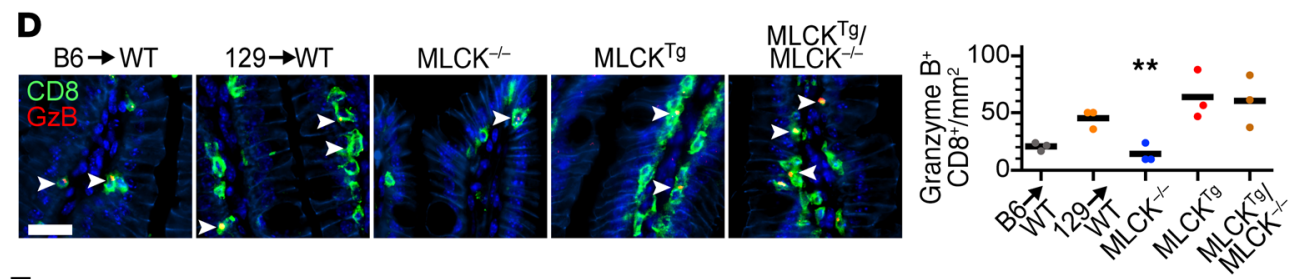

E
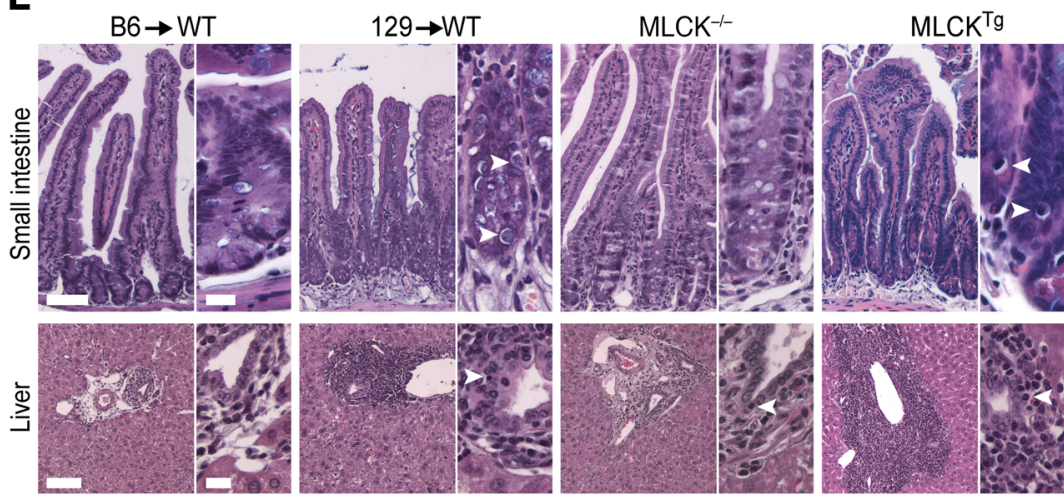

$\mathrm{MLCK}^{\mathrm{T}} / \mathrm{MLCK}^{-1-}$
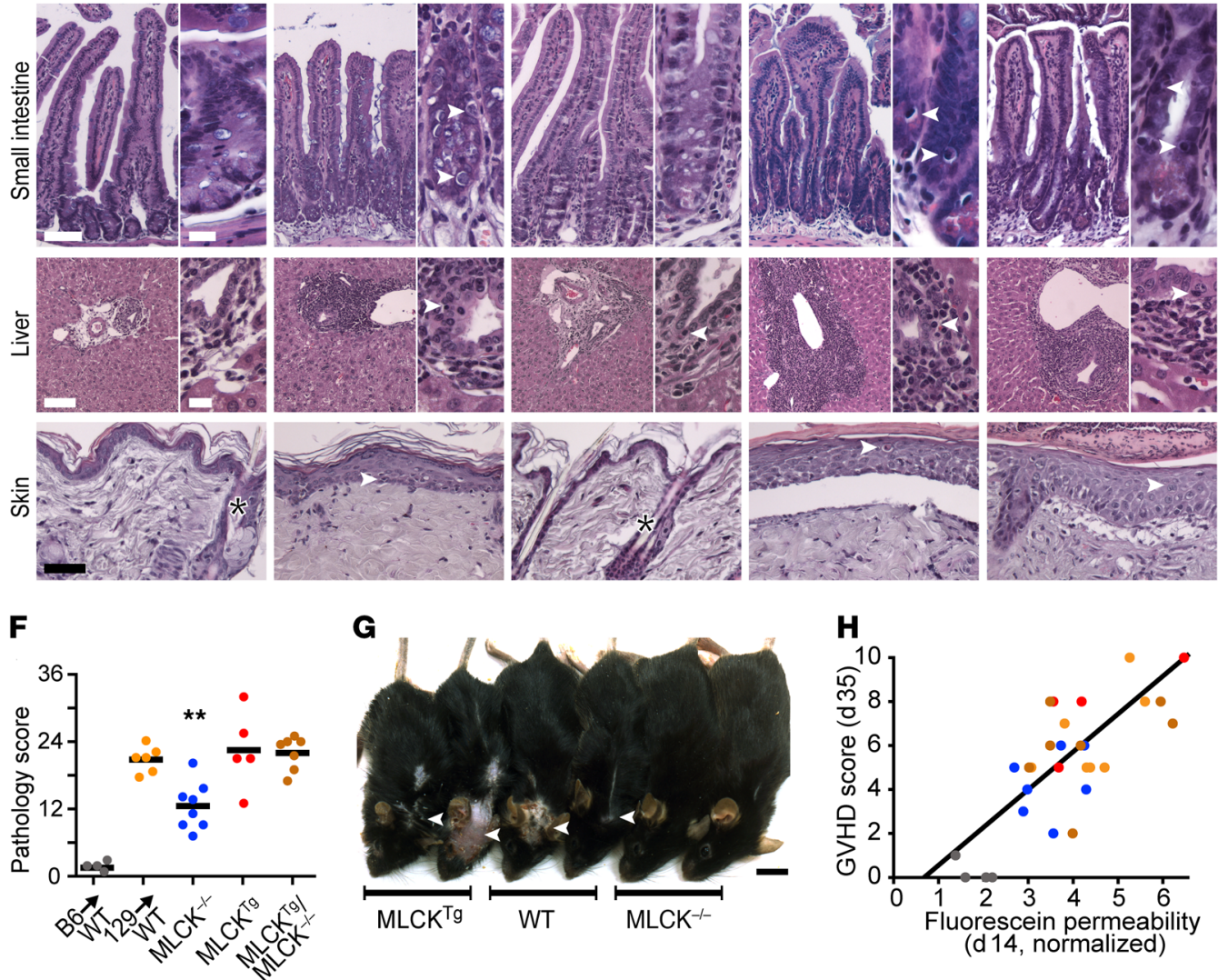

Figure 5. CVHD severity is modulated by intestinal epithelial MLCK210. B6 recipients of the indicated genotypes were lethally irradiated, followed by a 129 BMT. (A) Relative weight and (B) disease activity scores ( $n=6-9$ /group). ${ }^{*} P<0.05$, ANOVA with Bonferroni's correction vs. all other conditions. (C) Survival ( $n=8$-15/group). ${ }^{*} P<0.05$, Kaplan-Meier log-rank test, $\mathrm{MLCK}^{-1-}$ compared with all other groups. (D) Sections of jejunum were immunostained for CD8 (green) and granzyme B (red) 35 days after BMT. Representative images are shown. Arrowheads indicate colocalization. Scale bar: $20 \mu \mathrm{m}$. Each point represents an individual mouse. ${ }^{*} P<0.01$, ANOVA with Bonferroni's correction for MLCK210-KO mice vs. all other conditions (except syngeneic BMT) on d35. (E) Histopathology of jejunum (arrowheads denote apoptotic epithelial cells), liver (arrowheads denote lymphocytes infiltrating biliary epithelium), and skin (arrowheads denote apoptotic squamous cells, asterisks indicate preserved pilosebaceous units) on d35. Scale bars: intestine, $100 \mu \mathrm{m}, 20 \mu \mathrm{m}$; liver, $300 \mu \mathrm{m}, 50 \mu \mathrm{m} ; \mathrm{skin}$, $300 \mu \mathrm{m}$. (F) Total pathology scores (sum of jejunum, liver, and skin scores) 35 days after BMT. Each point represents an individual mouse. ${ }^{* *} P<0.01$, ANOVA with Bonferroni's correction (vs. all other conditions). (C) Gross photos of 2 mice/group 35 days after BMT. Arrowheads point to hair loss and skin ulcers. Scale bar: $1 \mathrm{~cm}$. (H) Correlation between intestinal permeability to fluorescein at 2 weeks after BMT and GVHD severity 5 weeks after BMT. Each point represents an individual mouse. Colors correspond to those for each condition shown in F. $r=0.75, P<0.01$ by Pearson's correlation coefficient and degrees of freedom. 
A

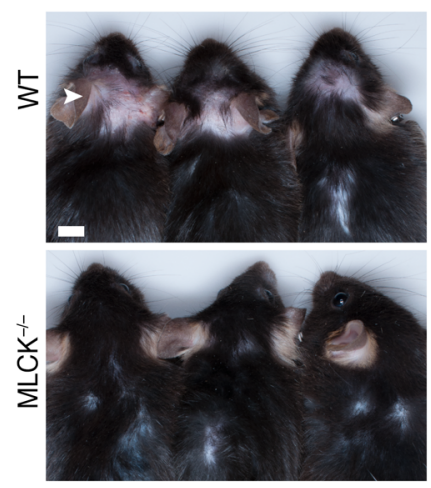

C

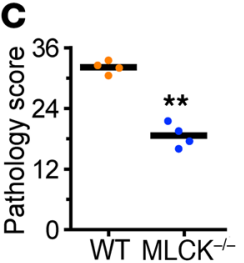

D

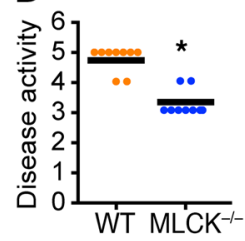

E
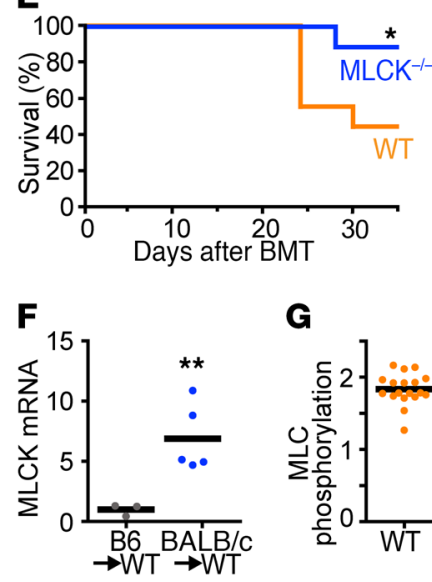

G

I

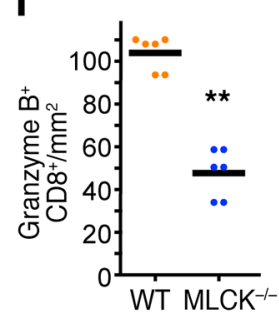

B

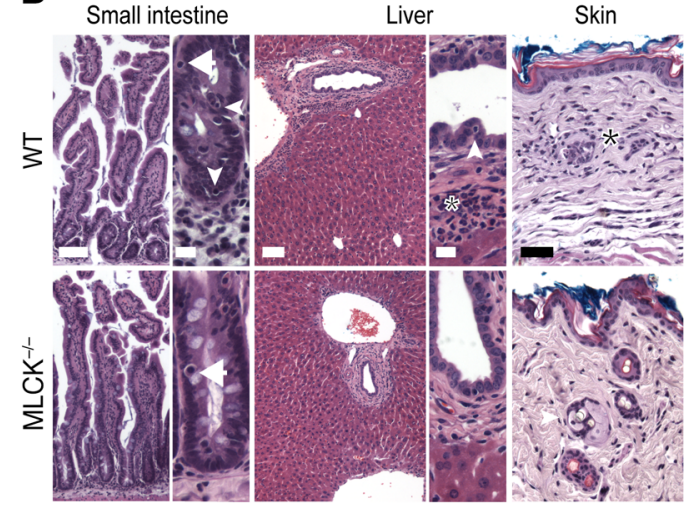

H
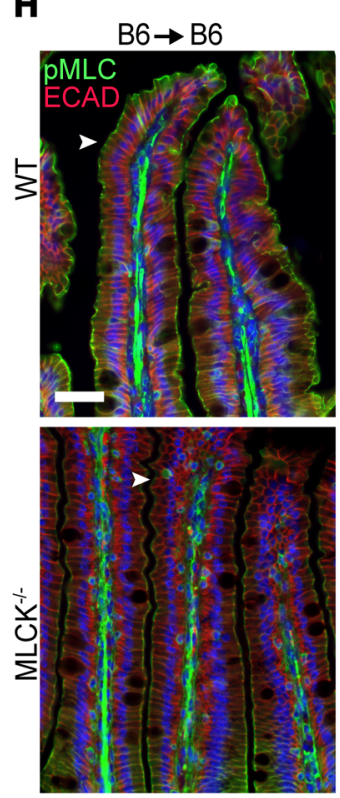

$\mathrm{BALB} / \mathrm{C} \rightarrow \mathrm{B} 6$
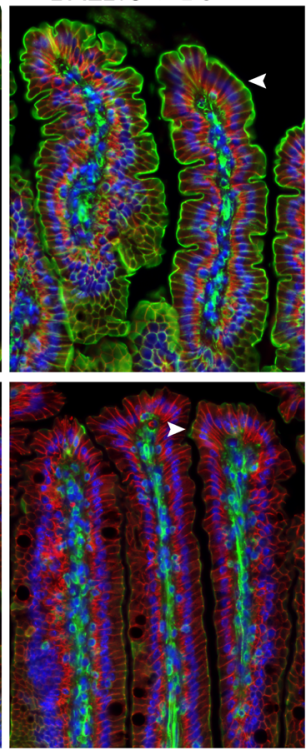

Figure 6. GVHD is diminished in $\mathrm{MLCK}^{-/-}$mice following major mismatch BMT. B6 WT or $\mathrm{MLCK}^{-1-}$ recipients were lethally irradiated, followed by BALB/C BMT. (A) Gross photos of 3 mice/group 35 days after BMT. Arrowhead points to hair loss and skin ulcers. Scale bar: $0.5 \mathrm{~cm}$. (B) Representative histopathology of jejunum (arrowheads denote apoptotic epithelial cells; arrows indicate intraepithelial lymphocytes), liver (arrowhead indicates intraepithelial lymphocyte; asterisk denotes neutrophils, i.e., active pericholangitis), and skin (asterisk is adjacent to a damaged pilosebaceous gland) on d35. Scale bars: intestine, $200 \mu \mathrm{m}, 20 \mu \mathrm{m}$; liver, $200 \mu \mathrm{m}, 20 \mu \mathrm{m}$; skin, $100 \mu \mathrm{m}$. (C) Total pathology scores (jejunum, liver, and skin) 35 days after BMT. Each point represents an individual mouse. ${ }^{* *} P<0.01$, 2-tailed $t$ test. (D) Disease activity scores at 35 days after BMT. Each point represents an individual mouse. ${ }^{*} P<0.01$, 2-tailed $t$ test. (E) Survival ( $n=9$ per group). ${ }^{*} P<0.05$, Kaplan-Meier log-rank test, MLCK $^{-1-}$ compared with WT. (F) MLCK210 mRNA was analyzed by qPCR in purified jejunal epithelial cells. Each point represents an individual mouse. Data are normalized to the mean of mice that did not receive BMT. ${ }^{*} P<0.01,2$-tailed $t$ test. ( $\mathbf{G}$ and $\mathbf{H}$ ) Jejunum was harvested 35 days after BMT and sections immunostained for phosphorylated myosin light chain (pMLC, green) and E-cadherin (ECAD, red). Arrowheads denote perijunctional actomyosin ring. ${ }^{* *} P<0.01,2$-tailed $t$ test. Scale bar: $25 \mu \mathrm{m}$. (I) Jejunum was harvested 35 days after BMT and immunostained for $C D 8$, granzyme $B$, and $\mathrm{E}$-cadherin. Left: quantification of $\mathrm{CD}^{+}$granzyme $\mathrm{B}^{+}$cells $/ \mathrm{mm}^{2}$ of tissue. ${ }^{* *} P<0.01$, 2 -tailed $t$ test. Right: representative images of CD8 (green) infiltration. Arrowheads in low-power views denote intraepithelial CD8 ${ }^{+}$ cells. Scale bars: $50 \mu \mathrm{m}, 10 \mu \mathrm{m}$.

The major advantage of the mOVA ${ }^{\mathrm{Tg}}$ GVHD model is that it allows direct analysis of the CD90.1+ OT-I T cells that drive disease. Based on reduced numbers of tissue-infiltrating $\mathrm{T}$ cells in MLCK $^{-1-}$ BMT recipients (Figure $4 \mathrm{G}$ and Figure $7 \mathrm{D}$ ), we expected that the proportion of CD90.1 $\mathrm{T}$ cells within lymph nodes and spleen would be similarly reduced in $\mathrm{MLCK}^{-/}$recipients. This was not, however, the case. The fractions of $\mathrm{CD}^{+} \mathrm{T}$ cells that were CD90.1 $1^{+}$in spleen, axillary/brachial lymph nodes, and mesenteric lymph nodes of $\mathrm{mOVA}^{\mathrm{Tg}}$ and $\mathrm{mOVA}^{\mathrm{Tg}} \mathrm{MLCK}^{-/}$BMT recipients were similar (Figure 7E). This suggested that some other characteristic of the CD90.1+ $\mathrm{T}$ cells might explain their enhanced patho- genicity in MLCK210-expressing BMT recipients. Consistent with this, the fraction of $\mathrm{CD} 90.1^{+} \mathrm{CD} 8^{+} \mathrm{T}$ cells expressing granzyme $\mathrm{B}$ was significantly greater in mesenteric lymph nodes of $\mathrm{mOVA}^{\mathrm{Tg}}$ BMT recipients relative to $\mathrm{mOVA}^{\mathrm{Tg}} \mathrm{MLCK}^{-/}$BMT recipients (Figure 7, F and G). Remarkably, this was also the case for polyclonal CD90.1-CD8 ${ }^{+} \mathrm{T}$ cells within mesenteric lymph nodes of $\mathrm{mOVA}^{\mathrm{Tg}}$ and $\mathrm{mOVA}^{\mathrm{Tg}} \mathrm{MLCK}^{-1-}$ BMT recipients (Figure 7, F and G). In contrast, the proportions of axillary/brachial lymph nodes and spleen CD 90.1 $1^{+}$and $\mathrm{CD} 90.1^{-} \mathrm{CD} 8^{+} \mathrm{T}$ cells expressing granzyme $\mathrm{B}$ were unaffected by MLCK210 deletion (Figure 7G). These data indicate that MLCK210 expression within the intestinal epithelium spe- 
A

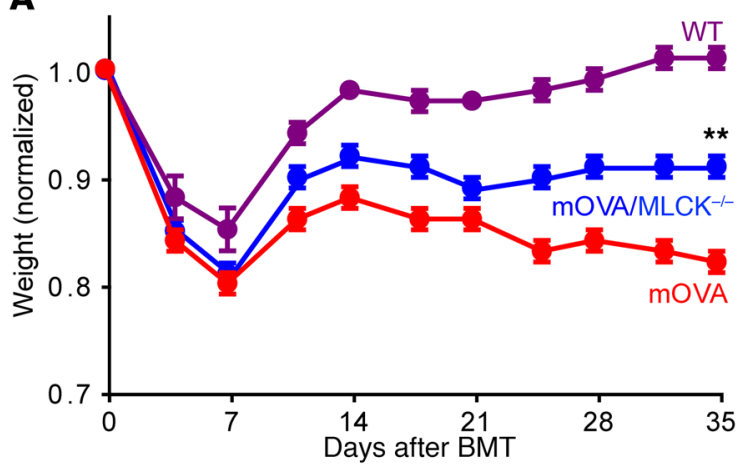

C
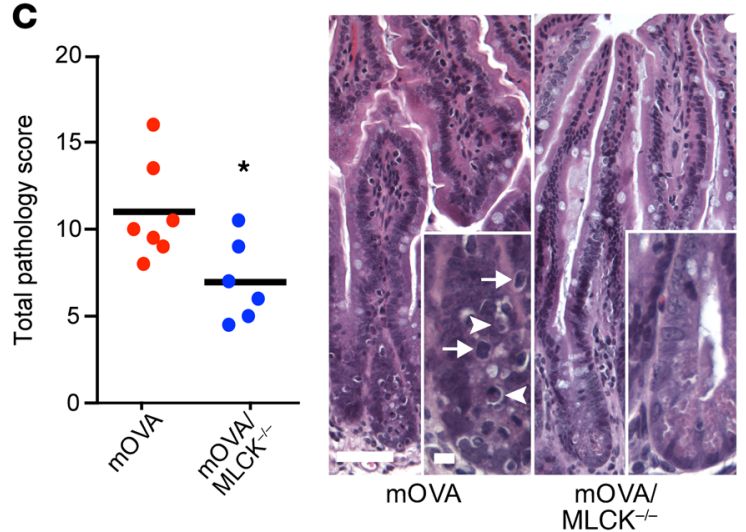

E

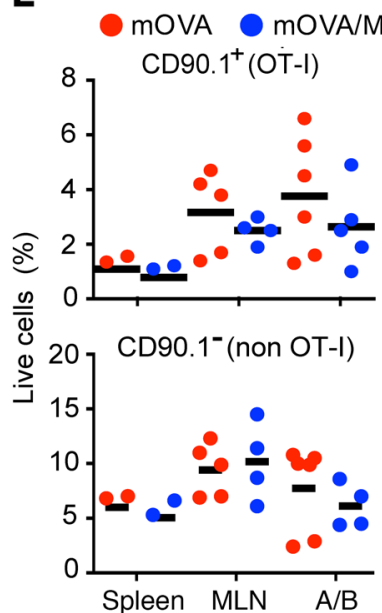

$\mathbf{F}$

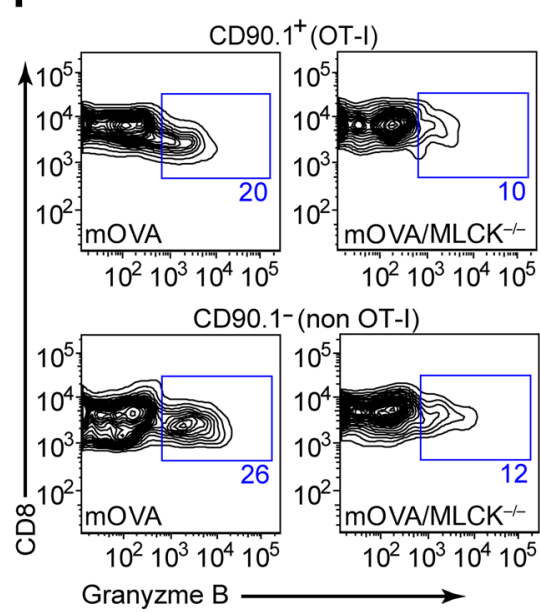

B

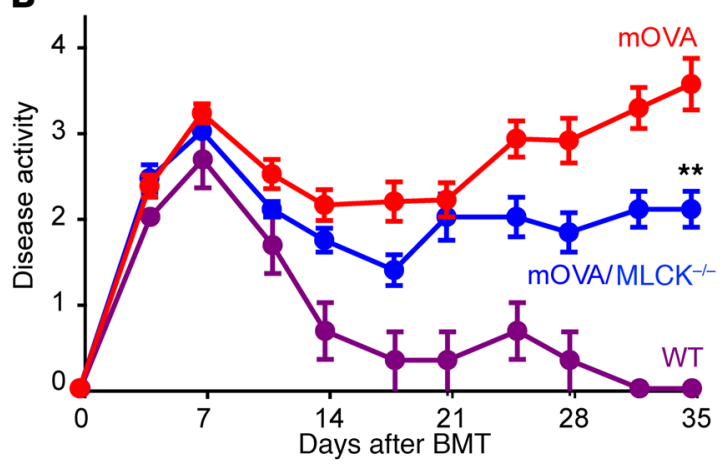

D

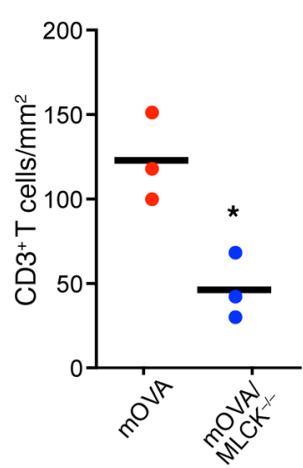

G
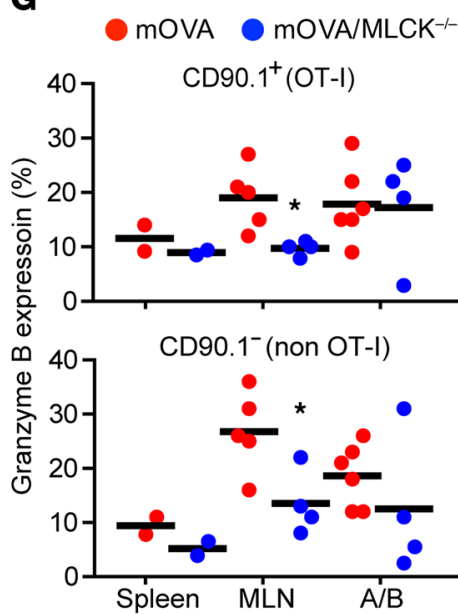

Figure 7. Genetic MLCK210 inhibition reduces effector CD8+ T cell accumulation within mesenteric, but not systemic, lymph nodes. B6 mOVA ${ }^{\mathrm{Tg}}(n=14)$, B6

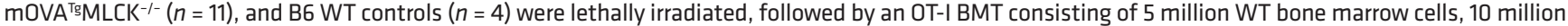
WT splenocytes, and 2 million OT-I splenocytes. (A) Weight and (B) disease activity scores. ${ }^{* *} P<0.01,2$-tailed $t$ tests on d35. (C) Pathology scores of GVHD target organs (jejunum, liver, and skin) and representative small intestinal histopathology 35 days after BMT. Each point represents an individual mouse. ${ }^{*} P<0.05$, 2-tailed $t$ test. Arrows denote intraepithelial lymphocytes; arrowheads denote apoptotic bodies. Scale bars: $50 \mu \mathrm{m}$; inset: $10 \mu \mathrm{m}$. (D) Small intestine samples from day 35 after BMT were immunostained for CD3 (green), ZO-1 (red), E-cadherin (ECAD, blue), and Hoechst 33342 (white). Representative images are shown. Scale bar: $50 \mu \mathrm{m}$. Graph shows $\mathrm{CD}^{+}$infiltration in the small intestine 35 days after BMT; each point represents an individual mouse. ${ }^{*} P<0.05$, 2-tailed $t$ test. (E) Flow cytometry analysis of cells isolated from spleen, mesenteric lymph nodes (MLN), and axillary/brachial lymph nodes (A/B) on d35 after BMT. The proportions of CD8 ${ }^{+} \mathrm{T}$ cells relative to live cells are shown. (F and $\left.\mathbf{C}\right)$ CD8 ${ }^{+} \mathrm{T}$ cell intracellular granzyme B expression as evaluated by flow cytometry 35 days after BMT. (F) Representative flow cytometry plots and (G) quantification. Each point represents an individual sample. ${ }^{*} P<0.05$, 2-tailed $t$ test.

cifically enhances the cytolytic potential of $\mathrm{CD} 8^{+}$effector $\mathrm{T}$ cells within regional lymph nodes and suggest that this may be a principal mechanism by which increased intestinal epithelial tight junction permeability drives systemic GVHD propagation.

\section{Discussion}

We show here that preventing MLCK-dependent intestinal epithelial tight junction barrier dysfunction can limit GVHD propagation, as defined by reduced tissue damage, cytolytic $\mathrm{T}$ cell 
differentiation, and mortality. The idea that tight junction preservation could be beneficial has long been proposed $(4,13$, $38,39)$, but the hypothesis has never been tested by selectively preventing disease-associated barrier loss during the propagation phase. Our use of mice lacking MLCK210 allowed us to determine the selective impact of cytoskeletally mediated intestinal epithelial tight junction permeability on GVHD and to distinguish this effect from pretransplant conditioningassociated damage during disease initiation. Our data demonstrate a causal role for MLCK210-dependent tight junction regulation, rather than gross epithelial damage, in driving GVHD propagation after recovery from conditioning-induced damage. The results further suggest that MLCK210-dependent changes in intestinal tight junction permeability promote disease propagation by establishing a local environment that fosters cytolytic effector $\mathrm{CD}^{+} \mathrm{T}$ cell expansion. We also observed reduced $\mathrm{CD}^{+} \mathrm{T}$ cell infiltration in the distant target organs of the liver and skin in $\mathrm{MLCK}^{--}$mice after allogeneic BMT compared with WT recipients. We speculate that the decrease in $\mathrm{CD}^{+} \mathrm{T}$ cells in systemic target organs in $\mathrm{MLCK}^{-/}$mice reflects quelling of the cytokine storm propagated by tight junction-mediated barrier dysfunction.

In addition to regulating intestinal epithelial tight junctions, MLCK210 has been reported to regulate endothelial retraction and apoptosis (40-43). Moreover, endothelial damage and increased microvascular permeability have been linked to GVHD pathogenesis (44-46). We therefore considered the alternative hypothesis that the protective effect of MLCK210 deletion could reflect preservation of vascular, rather than intestinal, barrier function. We tested this in two ways. First, we directly assessed vascular permeability to fluorescently tagged albumin. WT and $\mathrm{MLCK}^{-/-}$mice demonstrated similar loss of vascular albumin after BMT. The one qualitative difference was that albumin released from vessels within the villus highlighted sharply defined lateral intercellular spaces with a bright spot just beneath the apical junctional complex in $\mathrm{MLCK}^{-/}$, but not WT, mice. This suggests that albumin was able to leak out of vessels and across the epithelial basement membrane, but that diffusion was limited by the epithelial tight junction. Although vascular albumin was similarly lost in WT mice, fluorescent albumin within the intestinal epithelium of these mice created wider profiles of lateral intercellular spaces. These data indicate that vascular albumin leakage and barrier loss after BMT are similar but that intestinal epithelial barrier function is preserved during GVHD in $\mathrm{MLCK}^{-/}$, relative to WT, mice.

A potential contribution of endothelial MCLK210 was further probed using a genetic approach. Because tissue-specific MLCK210-knockout mice are not available, we took advantage of MLCK $^{\mathrm{Tg}}$ mice that express constitutively active MLCK under control of the intestinal epithelium-specific 9-kb villin promoter $(14,15,47)$. Expression of this transgene has been shown to restore colitis-associated MLC phosphorylation within intestinal epithelia of MLCK210-deficient mice (15). The transgene also restored GVHD in $\mathrm{MLCK}^{-1-}$ mice; the magnitude of disease was similar in WT, MLCK ${ }^{\mathrm{Tg}} \mathrm{MLCK}^{-/}$, and $\mathrm{MLCK}^{\mathrm{Tg}}$ mice; and severity in each was greater than that in $\mathrm{MLCK}^{-/-}$mice. Thus, intestinal epithelium-specific MLCK activation and consequent MLC phosphorylation are able to complement MLCK210 deletion. Since the transgene is not expressed in endothelium, the contrast between $\mathrm{MLCK}^{\mathrm{Tg}} \mathrm{MLCK}^{-/-}$and $\mathrm{MLCK}^{-/-}$mice indicates that endothelial MLCK210 is not required for GVHD propagation. This might be surprising, given the ability of MLCK210 to regulate endothelial permeability $(29,48-52)$. However, our data demonstrate that GVHD-associated increases in microvascular permeability are comparable in $\mathrm{WT}$ and $\mathrm{MLCK}^{-/-}$recipients. We must therefore conclude that other effectors (53-57) or direct endothelial damage $(40,44,46,58,59)$ are responsible for increased microvascular permeability in GVHD.

The primary clinical approach to treating GVHD after allogeneic BMT is immunosuppression (7). However, immunosuppression also increases risk of infection; compromises graft viability; and, in the case of HSCT as part of cancer therapy, can dampen graft-versus-tumor activity (60-66). Thus, there is an urgent need for alternate approaches to prevent GVHD development or reduce its severity. The current study identifies MLCK210 as a critical regulator of GVHD propagation and indicates that MLCK210 may be a promising therapeutic target. This will, however, require defining mechanisms to avoid toxicities resulting from inhibition of non-barrier functions of epithelial MLCK210 as well as smooth muscle MLCK, which has an identical catalytic domain $(15,67,68)$.

These studies clearly distinguish between GVHD initiation and propagation and indicate that MLCK210-dependent increases in intestinal epithelial tight junction permeability are central to propagation. In contrast, previous work from our group and others indicates that tight junction-independent barrier loss, due to epithelial damage, is necessary for GVHD initiation (4). Thus, while barrier loss is critical to both initiation and propagation of GVHD, the mechanisms and impact of barrier loss differ markedly.

Finally, these studies demonstrate that the magnitude of increases in intestinal permeability shortly after GVHD initiation, i.e., on d14, correlates with disease severity at later times. Noninvasive analyses of intestinal permeability are relatively easy to accomplish in patients $(69,70)$. It would therefore be of interest to determine whether intestinal permeability has similar prognostic value in patients. If so, one could envision developing barrier-restorative therapies that are used selectively in patients with increased intestinal permeability at early times after HSCT.

In summary, these data show that MLCK210-mediated intestinal epithelial barrier dysfunction is a primary contributor to systemic GVHD propagation. The results also suggest that targeting non-hematopoietic functions, such as epithelial barrier integrity, may provide an alternative to immunosuppression for the treatment of GVHD.

\section{Methods}

Immunohistochemistry and immunofluorescence. Deidentified patient biopsies were obtained from the archives of the University of Chicago Department of Pathology under an IRB-approved protocol. Duodenal biopsies from subjects with GVHD were included only if (i) they were collected 2 or more weeks after HSCT, in order to exclude effects of pretransplant conditioning or other interventions in the peritransplant period; and (ii) clinical and histological features of disease were present. Biopsies from patients who had not undergone HSCT and did not have duodenal disease served as controls. Formalin- 
fixed mouse tissues were embedded in paraffin and assembled into tissue microarrays before staining. After deparaffinization, $5-\mu \mathrm{m} \mathrm{sec}-$ tions were rehydrated, and antigen was unmasked by boiling in 0.01 citrate ( $\mathrm{pH}$ 6.0) or $0.01 \mathrm{M}$ Tris/EDTA ( $\mathrm{pH}$ 9.0), both with $0.05 \%$ Tween-20. Slides were stained with rat anti-human ZO-1 (clone 6B6E4), rat anti-mouse ZO-1 (clone R40.76), mouse anti-E-cadherin (Abcam, clone M168), rabbit anti- $\beta$-actin (Abcam, ab8227), rabbit antiphosphorylated MLC (Cell Signaling Technology, catalog 3671), mouse anti-MLCK (MilliporeSigma, clone K36), rat anti-CD3 (Bio-Rad, clone CD3-12), rabbit anti-CD3 (Abcam, clone SP7), biotinylated rat anti$\mathrm{CD} 8 \alpha$ (Invitrogen, clone 4SM15), biotinylated rat anti-CD4 (Invitrogen, clone 4SM95), rabbit anti-CD4 (Abcam, clone EPR19514), rabbit antigranzyme B (LifeSpan Biosciences, catalog LS-B7602), biotinylated rat anti-Foxp3 (Invitrogen, clone FJK-16s), or mouse anti-T-bet (Invitrogen, clone 4B10). MLCK detection in human samples used donkey antimouse IgG conjugated to HRP and the Envision+ System-HRP (DAB) kit (Dako). All other primary antibodies were detected using Alexa Fluor-conjugated streptavidin or highly purified secondary Fab' ${ }_{2}$ fragments (Jackson ImmunoResearch Laboratories Inc.) with Hoechst 33342 (Invitrogen). Stained sections were mounted in Permount for peroxidase stains and ProLong Diamond (Invitrogen) for fluorescent stains. H\&Estained and immunoperoxidase-stained tissues were imaged using a DMLB microscope (Leica) with 10 $\times$ HCFL PLAN NA0.25 and 40 $\times$ HCFL PLAN NA0.65 objectives and a MicroPublisher 3.3 CCD camera (QImaging) controlled by QCapture Pro 6. Post-acquisition processing was performed in Adobe Photoshop Lightroom. Fluorescence-stained tissues were imaged using either (i) a DM4000 epifluorescence microscope (Leica) equipped with 63× NA1.32 HCX PlanApo and 20× NA0.70 HC PlanApo objectives and Retiga Exi (QImaging) or Coolsnap HQ2 (Photometrics) cameras or (ii) an Axioplan 2 microscope (Zeiss) with 20× NA0.8 Plan-Apochromat and 100× NA1.30 Plan-Neofluar objectives and a Coolsnap HQ (Photometrics) camera. All fluorescence microscopes were controlled by Metamorph 7 (Molecular Devices). Images of larger tissue areas, e.g., for quantitative analysis of $\mathrm{T}$ cell infiltrates, were collected as tiled images using a DM4000, 20× NA0.70 HC PlanApo objective, and Coolsnap HQ2 controlled by custom journals with MetaMorph. Post-acquisition processing, including stitching of tiled images and quantitative analysis of signal intensity, was performed using Metamorph. T cell infiltrates were counted manually on tiled images.

Mice. Donor female 129 S6 and BALB/c mice were purchased from Taconic and the Jackson Laboratory, respectively. All mice were on a C57BL/6J (B6) background, and were bred and maintained at the University of Chicago, Brigham and Women's Hospital, or Boston Children's Hospital. B6 mice that express membrane-bound OVA on the surface of all cells (mOVA) were purchased from the Jackson Laboratory. B6 CD90.1/OT-I mice were generated from mice purchased from the Jackson Laboratory. MLCK210 ${ }^{-/-}$and MLCK ${ }^{\mathrm{Tg}}$ mice were developed as previously described $(14,29)$. MLCK210 ${ }^{-/}$mice (29) were backcrossed to $\mathrm{B} 6$ mice for 10 generations, and background was confirmed by high density SNP analyses.

Transplantations. For allogeneic BMT, $5 \times 10^{6}$ bone marrow cells and $30 \times 10^{6}$ million splenocytes (minor mismatch BMT) or $5 \times 10^{6}$ bone marrow cells and $10 \times 10^{6}$ million splenocytes (major mismatch $\mathrm{BMT})$ were given to age-matched, 7- to 12-week-old B6 recipients 24 hours after lethal irradiation (11 Gy). Antigen-specific GVHD was induced by i.v. injection of $5 \times 10^{6}$ bone marrow cells and $10 \times 10^{6}$ splenocytes from B6 WT mice and $2 \times 10^{6}$ B6 CD90.1/OT-I splenocytes into lethally irradiated $\mathrm{B} 6$ mOVA recipient mice. Mice were weighed and scored semiweekly. Disease activity was scored from 0 to 2 for posture, activity, fur texture, and hair loss, for a maximum score of 8. GVHD score was calculated by adding scores for weight loss relative to starting weight (>5\%: $1 ;>7.5 \%: 2 ;>10 \%: 3 ;>12.5 \%: 4 ;>15 \%: 5)$ and disease activity (activity $1-3: 1 ; 4: 2 ; 5: 3 ; 6: 4 ; 7-8: 5$ ) for a maximum total score of 10 . Mice were sacrificed and considered dead of disease, i.e., deceased, if weight loss was greater than $20 \%$ or disease activity was greater than 7 for two consecutive measurements, as required by IACUC policies.

Cytokine measurements. Serum or jejunal sections $(0.5 \mathrm{~cm})$ homogenized in cell lysis buffer (Bio-Rad) were assayed for IFN- $\gamma$, TNF, and IL-1 $\beta$ by ELISA using Ready-SET-Go! kits (eBioscience).

MLCK210 transcript quantification. For MLCK210 mRNA quantification, jejunal epithelial cells were isolated using Matrigel Cell Recovery Solution (Corning), as described previously (71, 72). RNA was extracted using the RNeasy mini KIT (QIAGEN). After DNase treatment (Invitrogen), cDNA was generated using iScript RT supermix (Bio-Rad). SsoAdvanced Universal SYBR Green Supermix (Bio-Rad), primers against long Mylk (GCGTGATCAGCCTGTTCTTTCTAA, GCCCCATCTGCCCTTCTTTGACC) and Krt8 (AAGGTGTGGAAAGAGCTAGCC, ATAGACGTTGACTGCACGCACT), and a CFX96 real-time thermocycler (Bio-Rad) were used for detection. Raw data were analyzed by the $\Delta \mathrm{Ct}$ method, with $\mathrm{Krt} 8 \mathrm{used}$ for normalization. Changes were calculated by the $\Delta \Delta \mathrm{Ct}$ method.

Intestinal and vascular permeability assays. Mice were denied access to food, but allowed water, for 3 hours before delivery of 12 mg FITC-4-kDa dextran or $0.25 \mathrm{mg}$ free (unconjugated) fluorescein by oral gavage. Serum was collected after 3 hours and analyzed using a SynergyHT platereader (Bio-Tek) with $485 \mathrm{~nm}$ excitation and $528 \mathrm{~nm}$ emission. Data were normalized to age and sex-matched disease-free controls. For vascular permeability, 200 $\mu \mathrm{g}$ Alexa-647 BSA (Invitrogen) in $200 \mu \mathrm{l}$ 0.9\% saline was injected i.v. 30 minutes before mice were sacrificed. Segments $(1.0 \mathrm{~cm})$ of jejunum were fixed in $4 \%$ paraformaldehyde in PBS overnight, equilibrated in $30 \%$ sucrose, and snap-frozen in optimal cutting temperature (OCT) media. Thick $(20 \mu \mathrm{m})$ cryosections were cut, mounted in SlowFade Diamond (Invitrogen), and imaged using a Leica DMI6000 inverted spinning disk (Yokogawa CSU-X1) confocal microscope with a 20× NA0.70 HC PlanApo objective and a Zyla 4.2 sCMOS camera (Andor) camera. Regions demonstrating well-oriented mucosal architecture, defined villi and crypts, and a visible vascular core within the villus were imaged as $15-\mu \mathrm{m}$-thick $Z$-stacks at 0.5 - $\mu \mathrm{m}$ intervals. Sum projections were created using Metamorph and vascular dye retention calculated as mean intensity of the vascular core. Matched pseudocolor scales were applied for image presentation.

Histopathology scoring. Histopathological analysis of GVHD target organs was performed by a subspecialty-trained gastrointestinal pathologist blinded to the experimental conditions. Jejunum, liver, and skin were scored from 0 to 3 at 0.5 intervals based on 4 features, for a maximum organ score of 12 and a maximum total score of 36 using previously reported criteria (4).

Flow cytometry. Freshly isolated samples from lymph nodes and spleen were stained using PE-conjugated anti-CD90.1, FITCconjugated anti-CD8, APC-conjugated anti-V $\alpha 2$ (eBioscience), and BV510s-conjugated anti-granzyme B (BD). Flow cytometry was performed with a FACSCanto (BD) and analyzed with FlowJo. 
Statistics. All data presented are mean \pm SEM. Statistical significance was determined by unpaired, 2-tailed $t$ test, ANOVA with Bonferroni's correction, 2-tailed Mann-Whitney $U$ test, or Kaplan-Meier log-rank test. $P$ values and the type of statistical analysis performed are described in the figure legends. In figures, ${ }^{*} \mathrm{P}<$ 0.05 and ${ }^{* *} P<0.01 . P<0.05$ was considered significant.

Study approval. Studies of deidentified patient biopsies were approved by the University of Chicago Institutional Review Board. All experiments using mice were approved by and complied with regulations of the IACUCs of the University of Chicago, Brigham and Women's Hospital, or Boston Children's Hospital.

\section{Author contributions}

The project was conceived by SCN, PAS, and JRT. SCN, LZ, MLDMO, GS, AMW, WC, MCM, APS, KLE, GJB, NEJ, and JRT performed exper- iments. The manuscript was written and revised by SCN, PAS, and JRT with input from all authors. Figures were prepared by SCN and JRT.

\section{Acknowledgments}

We would like to thank the University of Chicago Human Tissue Resource Center for their expertise and assistance in recovery and IHC staining of human GVHD biopsies. This work was supported by NIH grants R01DK61931 (JRT), R01DK68271 (JRT), R24DK099803 (JRT), and R01AI110507 (PAS), and the Harvard Digestive Disease Center (P30DK034854).

Address correspondence to: Jerrold R. Turner, Brigham and Women's Hospital, Harvard Medical School, 77 Avenue Louis Pasteur, HNRB 730B, Boston, Massachusetts, USA. Phone: 617.525.8165; Email: jrturner@bwh.harvard.edu.
1. Ferrara JL, Smith CM, Sheets J, Reddy P, Serody JS. Altered homeostatic regulation of innate and adaptive immunity in lower gastrointestinal tract GVHD pathogenesis. J Clin Invest. 2017;127(7):2441-2451.

2. Clayburgh DR, et al. Epithelial myosin light chain kinase-dependent barrier dysfunction mediates $\mathrm{T}$ cell activation-induced diarrhea in vivo. J Clin Invest. 2005;115(10):2702-2715.

3. Mehta PA, et al. Radiation-free, alternativedonor HCT for Fanconi anemia patients: results from a prospective multi-institutional study. Blood. 2017;129(16):2308-2315.

4. Nalle SC, et al. Recipient NK cell inactivation and intestinal barrier loss are required for MHC-matched graft-versus-host disease. Sci Transl Med. 2014;6(243):243ra87.

5. Chen X, Dodge J, Komorowski R, Drobyski WR. A critical role for the retinoic acid signaling pathway in the pathophysiology of gastrointestinal graft-versus-host disease. Blood. 2013;121(19):3970-3980.

6. de Mooij CEM, Netea MG, van der Velden WJFM, Blijlevens NMA. Targeting the interleukin-1 pathway in patients with hematological disorders. Blood. 2017;129(24):3155-3164.

7. Cutler CS, Koreth J, Ritz J. Mechanistic approaches for the prevention and treatment of chronic GVHD. Blood. 2017;129(1):22-29.

8. Lounder DT, et al. Lower levels of vitamin A are associated with increased gastrointestinal graft-versus-host disease in children. Blood. 2017;129(20):2801-2807.

9. Carpenter PA. Vitamin A to reduce gut leak and GVHD? Blood. 2017;129(20):2715-2717.

10. Brown GR, Lindberg G, Meddings J, Silva M, Beutler B, Thiele D. Tumor necrosis factor inhibitor ameliorates murine intestinal graft-versus-host disease. Gastroenterology. 1999;116(3):593-601.

11. Noth R, et al. Increased intestinal permeability and tight junction disruption by altered expression and localization of occludin in a murine graft versus host disease model. BMC Gastroenterol. 2011;11:109.

12. Hanash AM, et al. Interleukin-22 protects intestinal stem cells from immune-mediated tissue damage and regulates sensitivity to graft versus host disease. Immunity. 2012;37(2):339-350.
13. Johansson JE, Ekman T. Gut toxicity during hemopoietic stem cell transplantation may predict acute graft-versus-host disease severity in patients. Dig Dis Sci. 2007;52(9):2340-2345.

14. Su L, et al. Targeted epithelial tight junction dysfunction causes immune activation and contributes to development of experimental colitis. Gastroenterology. 2009;136(2):551-563.

15. Su L, et al. TNFR2 activates MLCK-dependent tight junction dysregulation to cause apoptosismediated barrier loss and experimental colitis. Gastroenterology. 2013;145(2):407-415.

16. Blair SA, Kane SV, Clayburgh DR, Turner JR. Epithelial myosin light chain kinase expression and activity are upregulated in inflammatory bowel disease. Lab Invest. 2006;86(2):191-201.

17. Fegan $\mathrm{C}$, Poynton $\mathrm{CH}$, Whittaker JA. The gut mucosal barrier in bone marrow transplantation. Bone Marrow Transplant. 1990;5(6):373-377.

18. Rosenblatt J, Raff MC, Cramer LP. An epithelial cell destined for apoptosis signals its neighbors to extrude it by an actin- and myosin-dependent mechanism. Curr Biol. 2001;11(23):1847-1857.

19. Marchiando AM, et al. The epithelial barrier is maintained by in vivo tight junction expansion during pathologic intestinal epithelial shedding. Gastroenterology.2011;140(4):1208-1218.e1.

20. Madara JL. Maintenance of the macromolecular barrier at cell extrusion sites in intestinal epithelium: physiological rearrangement of tight junctions. JMembr Biol. 1990;116(2):177-184.

21. Wang F, Graham WV, Wang Y, Witkowski ED, Schwarz BT, Turner JR. Interferon-gamma and tumor necrosis factor-alpha synergize to induce intestinal epithelial barrier dysfunction by up-regulating myosin light chain kinase expression. Am J Pathol. 2005;166(2):409-419.

22. Graham WV, et al. Tumor necrosis factorinduced long myosin light chain kinase transcription is regulated by differentiation-dependent signaling events. Characterization of the human long myosin light chain kinase promoter. J Biol Chem. 2006;281(36):26205-26215.

23. Al-Sadi R, Ye D, Dokladny K, Ma TY. Mechanism of IL-1beta-induced increase in intestinal epithelial tight junction permeability. J Immunol. 2008;180(8):5653-5661.

24. Turner JR, et al. Physiological regulation of epi- thelial tight junctions is associated with myosin light-chain phosphorylation. Am J Physiol. 1997;273(4 Pt 1):C1378-C1385.

25 . Shen L, et al. Myosin light chain phosphorylation regulates barrier function by remodeling tight junction structure. J Cell Sci. 2006;119(Pt 10):2095-2106.

26. Weber CR, et al. Epithelial myosin light chain kinase activation induces mucosal interleukin-13 expression to alter tight junction ion selectivity. J Biol Chem. 2010;285(16):12037-12046.

27. Anderson JM, Van Itallie CM. Physiology and function of the tight junction. Cold Spring Harb Perspect Biol. 2009;1(2):a002584.

28. Turner JR. Intestinal mucosal barrier function in health and disease. Nat Rev Immunol. 2009;9(11):799-809.

29. Wainwright MS, et al. Protein kinase involved in lung injury susceptibility: evidence from enzyme isoform genetic knockout and in vivo inhibitor treatment. Proc Natl Acad Sci US A. 2003;100(10):6233-6238.

30. Madara JL. Intestinal absorptive cell tight junctions are linked to cytoskeleton. Am J Physiol. 1987;253(1 pt 1):C171-C175.

31. Katoh K, Kano Y, Amano M, Onishi H, Kaibuchi K, Fujiwara K. Rho-kinase--mediated contraction of isolated stress fibers. J Cell Biol. 2001;153(3):569-584.

32. Garcia JG, Davis HW, Patterson CE. Regulation of endothelial cell gap formation and barrier dysfunction: role of myosin light chain phosphorylation. JCell Physiol. 1995;163(3):510-522.

33. Garcia JG, Verin AD, Herenyiova M, English D. Adherent neutrophils activate endothelial myosin light chain kinase: role in transendothelial migration. J Appl Physiol. 1998;84(5):1817-1821.

34. Eutamene H, et al. LPS-induced lung inflammation is linked to increased epithelial permeability: role of MLCK. Eur Respir J. 2005;25(5):789-796.

35. el Marjou F, et al. Tissue-specific and inducible Cre-mediated recombination in the gut epithelium. Genesis. 2004;39(3):186-193.

36. Ehst BD, Ingulli E, Jenkins MK. Development of a novel transgenic mouse for the study of interactions between CD 4 and CD8 T cells during graft rejection. Am J Transplant. 2003;3(11):1355-1362.

37. Hogquist KA, Jameson SC, Heath WR, Howard 
JL, Bevan MJ, Carbone FR. T cell receptor antagonist peptides induce positive selection. Cell. 1994;76(1):17-27.

38. Hill GR, Ferrara JL. The primacy of the gastrointestinal tract as a target organ of acute graftversus-host disease: rationale for the use of cytokine shields in allogeneic bone marrow transplantation. Blood. 2000;95(9):2754-2759.

39. Nalle SC, Turner JR. Intestinal barrier loss as a critical pathogenic link between inflammatory bowel disease and graft-versus-host disease. Mucosal Immunol. 2015;8(4):720-730.

40. Petrache I, Verin AD, Crow MT, Birukova A, Liu F, Garcia JG. Differential effect of MLC kinase in TNF-alpha-induced endothelial cell apoptosis and barrier dysfunction. Am J Physiol Lung Cell Mol Physiol. 2001;280(6):L1168-L1178.

41. Xu C, Wu X, Hack BK, Bao L, Cunningham PN. TNF causes changes in glomerular endothelial permeability and morphology through a Rho and myosin light chain kinase-dependent mechanism. Physiol Rep. 2015;3(12):e12636.

42. Goeckeler ZM, Wysolmerski RB. Myosin light chain kinase-regulated endothelial cell contraction: the relationship between isometric tension, actin polymerization, and myosin phosphorylation. J Cell Biol. 1995;130(3):613-627.

43. Adderley SP, Zhang XE, Breslin JW. Involvement of the $\mathrm{H} 1$ histamine receptor, p38 MAP kinase, myosin light chains kinase, and Rho/ROCK in histamine-induced endothelial barrier dysfunction. Microcirculation. 2015;22(4):237-248.

44. Biedermann BC, et al. Endothelial injury mediated by cytotoxic $\mathrm{T}$ lymphocytes and loss of microvessels in chronic graft versus host disease. Lancet. 2002;359(9323):2078-2083.

45. Dumler JS, Beschorner WE, Farmer ER, Di Gennaro KA, Saral R, Santos GW. Endothelial-cell injury in cutaneous acute graft-versus-host disease. Am J Pathol.1989;135(6):1097-1103.

46. Schmid PM, et al. Endothelial dysfunction and altered mechanical and structural properties of resistance arteries in a murine model of graftversus-host disease. Biol Blood Marrow Transplant. 2014;20(10):1493-1500.

47. Edelblum KL, et al. The microbiome activates CD4 T-cell-mediated immunity to compensate for increased intestinal permeability. Cell Mol Gastroenterol Hepatol. 2017;4(2):285-297.

48. Sheldon R, Moy A, Lindsley K, Shasby S, Shasby
DM. Role of myosin light-chain phosphorylation in endothelial cell retraction. Am J Physiol. 1993;265(6 Pt 1):L606-L612.

49. Ohlmann P, et al. Deletion of MLCK210 induces subtle changes in vascular reactivity but does not affect cardiac function. Am J Physiol Heart Circ Physiol. 2005;289(6):H2342-H2349.

50. Moitra J, et al. A transgenic mouse with vascular endothelial over-expression of the non-muscle myosin light chain kinase- 2 isoform is susceptible to inflammatory lung injury: role of sexual dimorphism and age. Transl Res. 2008;151(3):141-153.

51. Moy AB, Shasby SS, Scott BD, Shasby DM. The effect of histamine and cyclic adenosine monophosphate on myosin light chain phosphorylation in human umbilical vein endothelial cells. J Clin Invest. 1993;92(3):1198-1206.

52. Wang $\mathrm{T}$, et al. Nonmuscle myosin light chain kinase regulates murine asthmatic inflammation. Am J Respir Cell Mol Biol. 2014;50(6):1129-1135.

53. Wójciak-Stothard B, Entwistle A, Garg R, Ridley AJ. Regulation of TNF-alpha-induced reorganization of the actin cytoskeleton and cell-cell junctions by Rho, Rac, and Cdc 42 in human endothelial cells. J Cell Physiol. 1998;176(1):150-165.

54. Garcia JG, et al. Sphingosine 1-phosphate promotes endothelial cell barrier integrity by Edg-dependent cytoskeletal rearrangement. JClin Invest. 2001;108(5):689-701.

55. Birukova AA, et al. Microtubule disassembly induces cytoskeletal remodeling and lung vascular barrier dysfunction: role of Rho-dependent mechanisms. JCell Physiol. 2004;201(1):55-70.

56. Siddiqui MR, et al. Caveolin-1-eNOS signaling promotes p190RhoGAP-A nitration and endothelial permeability. JCell Biol. 2011;193(5):841-850.

57. Yu Y, et al. Deletion of myosin light chain kinase in endothelial cells has a minor effect on the lipopolysaccharide-induced increase in microvascular endothelium permeability in mice. FEBS J. 2012;279(8):1485-1494.

58. Nürnberger W, Michelmann I, Burdach S, Göbel U. Endothelial dysfunction after bone marrow transplantation: increase of soluble thrombomodulin and PAI-1 in patients with multiple transplant-related complications. Ann Hematol. 1998;76(2):61-65.

59. Zelic M, et al. RIP kinase 1-dependent endothelial necroptosis underlies systemic inflam- matory response syndrome. J Clin Invest. 2018;128(5):2064-2075.

60. Ferrara JL. Novel strategies for the treatment and diagnosis of graft-versus-host-disease. Best Pract Res Clin Haematol. 2007;20(1):91-97.

61. Brennan TV, Yang Y. PD-L1 serves as a double agent in separating GVL from GVHD. JClin Invest. 2017;127(5):1627-1630.

62. Couturier M, et al. IL-22 deficiency in donor T cells attenuates murine acute graft-versus-host disease mortality while sparing the graft-versus-leukemia effect. Leukemia. 2013;27(7):1527-1537.

63. Veenstra RG, et al. B7-H3 expression in donor $\mathrm{T}$ cells and host cells negatively regulates acute graft-versus-host disease lethality. Blood. 2015;125(21):3335-3346.

64. Ramadan A, et al. Specifically differentiated $\mathrm{T}$ cell subset promotes tumor immunity over fatal immunity. J Exp Med. 2017;214(12):3577-3596.

65. van Bergen CA, et al. Selective graft-versusleukemia depends on magnitude and diversity of the alloreactive $\mathrm{T}$ cell response. J Clin Invest. 2017;127(2):517-529.

66. Zeng Y, Stokes J, Hahn S, Hoffman E, Katsanis E. Activated MHC-mismatched T helper-1 lymphocyte infusion enhances GvL with limited GvHD. Bone Marrow Transplant. 2014;49(8):1076-1083.

67. He WQ, et al. Myosin light chain kinase is central to smooth muscle contraction and required for gastrointestinal motility in mice. Gastroenterology. 2008;135(2):610-620.

68. Russo JM, et al. Distinct temporal-spatial roles for rho kinase and myosin light chain kinase in epithelial purse-string wound closure. Gastroenterology. 2005;128(4):987-1001.

69. Smecuol E, et al. Acute gastrointestinal permeability responses to different non-steroidal antiinflammatory drugs. Gut. 2001;49(5):650-655.

70. Meddings JB, Gibbons I. Discrimination of site-specific alterations in gastrointestinal permeability in the rat. Gastroenterology. 1998;114(1):83-92.

71. Tsai PY, et al. IL-22 Upregulates Epithelial Claudin-2 to Drive Diarrhea and Enteric Pathogen Clearance. Cell Host Microbe. 2017;21(6):671-681.e4.

72. Nik AM, Carlsson P. Separation of intact intestinal epithelium from mesenchyme. BioTechniques. 2013;55(1):42-44. 